\title{
EpiCurator: an immunoinformatic workflow to predict and prioritize SARS-CoV-2 epitopes
}

\author{
Cristina S Ferreira ${ }^{1}$, Yasmmin C Martins $^{1}$, Rangel Celso Souza $^{1}$, Ana Tereza R Vasconcelos ${ }^{\text {Corresp. } 1}$ \\ ${ }^{1}$ Bioinformatics Laboratory, National Laboratory of Scientific Computation, Petrópolis, Rio de Janeiro, Brazil \\ Corresponding Author: Ana Tereza R Vasconcelos \\ Email address: atrv@Incc.br
}

The ongoing coronavirus 2019 (COVID-19) pandemic, triggered by the emerging SARSCoV-2 virus, represents a global public health challenge. Therefore, the development of effective vaccines is an urgent need to prevent and control virus spread. One of the vaccine production strategies uses the in silico epitope prediction from the virus genome by immunoinformatic approaches, which assist in selecting candidate epitopes for in vitro and clinical trials research. This study introduces the EpiCurator workflow to predict and prioritize epitopes from SARS-CoV-2 genomes by combining a series of computational filtering tools. To validate the workflow effectiveness, SARS-CoV-2 genomes retrieved from the GISAID database were analyzed. We identified 11 epitopes in the receptor-binding domain (RBD) of Spike glycoprotein, an important antigenic determinant not previously described in the literature or published on the Immune Epitope Database (IEDB). Interestingly, these epitopes have a combination of important properties: recognized in sequences of the current variants of concern, present high antigenicity, conservancy, and broad population coverage. The RBD epitopes were the source for a multi-epitope design to in silico validation of their immunogenic potential. The multi-epitope overall quality was computationally validated, endorsing its efficiency to trigger an effective immune response since it has stability, high antigenicity and strong interactions with Toll-Like Receptors (TLR). Taken together, the findings in the current study demonstrated the efficacy of the workflow for epitopes discovery, providing target candidates with immunogen development. 
1 EpiCurator: an immunoinformatic workflow to predict 2 and prioritize SARS-CoV-2 epitopes

3

4

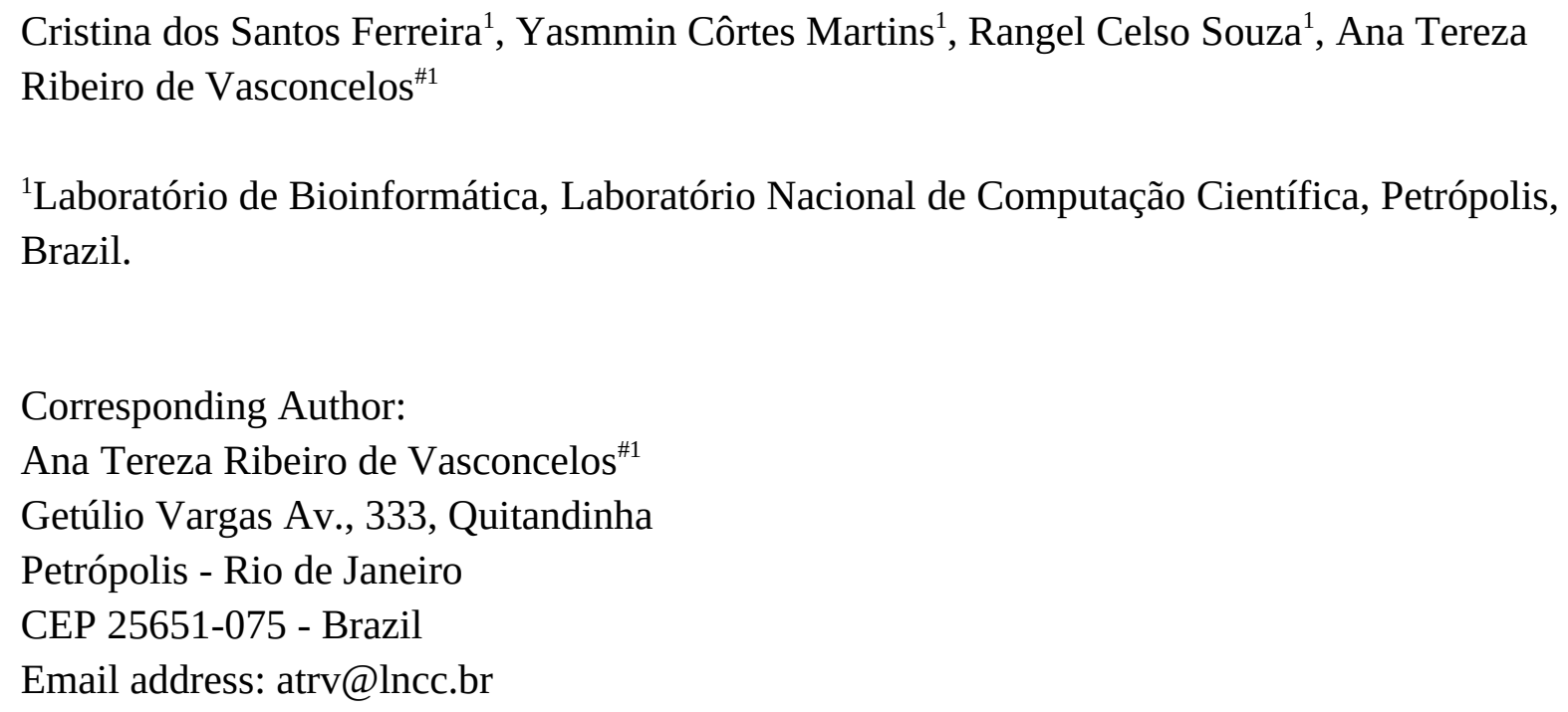

\section{Abstract}

The ongoing coronavirus 2019 (COVID-19) pandemic, triggered by the emerging SARS-CoV-2 virus, represents a global public health challenge. Therefore, the development of effective vaccines is an urgent need to prevent and control virus spread. One of the vaccine production strategies uses the in silico epitope prediction from the virus genome by immunoinformatic approaches, which assist in selecting candidate epitopes for in vitro and clinical trials research. This study introduces the EpiCurator workflow to predict and prioritize epitopes from SARS$\mathrm{CoV}-2$ genomes by combining a series of computational filtering tools. To validate the workflow effectiveness, SARS-CoV-2 genomes retrieved from the GISAID database were analyzed. We identified 11 epitopes in the receptor-binding domain (RBD) of Spike glycoprotein, an important antigenic determinant not previously described in the literature or published on the Immune Epitope Database (IEDB). Interestingly, these epitopes have a combination of important properties: recognized in sequences of the current variants of concern, present high antigenicity, conservancy, and broad population coverage. The RBD epitopes were the source for a multiepitope design to in silico validation of their immunogenic potential. The multi-epitope overall quality was computationally validated, endorsing its efficiency to trigger an effective immune response since it has stability, high antigenicity and strong interactions with Toll-Like Receptors (TLR). Taken together, the findings in the current study demonstrated the efficacy of the workflow for epitopes discovery, providing target candidates with immunogen development.

\section{Introduction}

The emergence of the SARS-CoV-2 infection, causing COVID-19 disease, has spread rapidly worldwide and represents a global challenge for public health (Cohen \& Normile, 2020; 
42 Chakraborty et al., 2020). This virus was first reported in Wuhan in December 2019 (Huang et

43 al., 2020) and quickly evolved, with the emergence of several variants (Cella et al., 2021;

44 Brüssow, 2021). According to the World Health Organization (WHO) classifications, there are

45 four variants of concern (VOCs) currently spread worldwide designated as Alpha (B.1.1.7), Beta

46 (B.1.351), Gamma (P.1), and Delta (B.1.617.2) (Faria et al., 2020; Rambaut et al., 2020; WHO,

47 2021; Tegally et al., 2021). In addition, the VOCs are characterized by the possibility to impact

48 the disease severity, such as the possible increased risk of hospitalization, mortality, and

49 capability of evading vaccination-induced immune response (Prévost \& Finzi, 2021; Geers et al.,

50 2021). The constant emergence of new variants keeps the contagiousness of SARS-CoV-2, which

51 increases the uncertainty of virus spread (Brüssow, 2021; Naveca et al., 2021).

52 Current research aims to develop effective interventions for controlling and preventing the

53 COVID-19 pandemic, furthermore, vaccination is still the most economical and effective

54 approach to prevent infection by the virus (Shang et al., 2020). At the time of writing, 22

55 vaccines against SARS-CoV-2 have been approved by at least one country (McGill COVID19

56 Vaccine Tracker Team, 2021). Five vaccines were approved for emergency use authorization and listed by WHO Emergency Use Listing (EUL) (Bulla, 2021; Mascellino et al., 2021). The Pfizer/ BioNTech vaccine is based on messenger RNA (mRNA), coding for viral spike (S) proteins (Badiani et al., 2020). The Moderna vaccine is a lipid nano-particle-encapsulated mRNA-based vaccine that encodes a full-length spike (Mahase, 2020). The Johnson \& Johnson (Janssen) is a recombinant, non-replicating adenovirus vector encoding a full-length S protein (Livingston, Malani \& Creech, 2021). The Oxford-AstraZeneca is a chimpanzee adenovirus vectored DNA vaccine (Knoll \& Wonodi, 2021), and the CoronaVac is an inactivated virus COVID-19 (Gao et al., 2020; Mallapaty, 2021). production (Thanh Le et al., 2020; Siracusano, Pastori \& Lopalco, 2020; Yoshida et al., 2021). However, several studies report the capability of a new mutation interrupting the binding with some neutralizing antibodies (Greaney et al., 2021; Hoffmann et al., 2021; Andreano et al., 2021), which could clarify the spread of the current VOCs. The main reasons for this capability are the several changes (mutation and deletion) in the S protein, which is responsible by the increased affinity between the receptor-binding domain (RBD) and the human cellular receptor angiotensin-converting enzyme 2 (ACE2), promoting the antibodies escape (Thomson et al., 2021; Greaney et al., 2021; Liu et al., 2021; Rotondo et al., 2021). cooperation, and innovation have contributed to the accelerated development of COVID-19 vaccines (Bloom et al., 2021). These efforts lead various researchers to carry out diversified methodologies of vaccine design such as a peptide-based vaccine, virus-like particle, replicating and non-replicating viral vectors, DNA or RNA, live attenuated virus, recombinant designed proteins, nanoparticles vaccine, and inactivated virus (Medhi et al., 2020; Krammer, 2020; Di Natale et al., 2020; Kyriakidis et al., 2021; Shahcheraghi et al., 2021). 
84 four from 22 approved vaccines have a peptide-based design (McGill COVID19 Vaccine Tracker

85 Team, 2021). The advantages of peptide-based vaccines include their capability to target very

86 specific epitopes decreasing the risks associated with allergic and autoimmune responses, besides

87 involving minimal viral components to stimulate adaptive immunity (Di Natale et al., 2020).

88 Additionally, their chemical or recombinant cloning synthesis allows large-scale production with

89 low costs and high reproducibility (Sun, 2013; Skwarczynski \& Toth, 2016; Hudu, Shinkafi \&

90 Umar, 2016).

91 The peptide-based vaccine design requires the immunoinformatic approach as part of the

92 computational vaccinology strategy for epitopes prediction (Ramana \& Mehla, 2020; Oli et al.,

93 2020; Lu et al., 2021). This approach regards the wide availability of the SARS-CoV-2 NGS

94 (Next-Generation Sequencing) information associated with human leukocyte antigen (HLA)

95 profile (Kazi et al., 2018; Oli et al., 2020; Sharma et al., 2020) to identify T cell epitopes.

96 Therefore, these epitopes have the capability to effectively bind to HLA molecules activating a

97 long-lasting immune response mediated by CD8+ and CD4+ T cells (Fast, Altman \& Chen, 2020;

98 Wang \& Gui, 2020).

99 T cell epitopes offer advantages for vaccine design since it does not depend on the recognition of

100 structural proteins (Bashir et al., 2021; Redd et al., 2021) and are less affected by deletions and

101 mutations of emergent variants (Ribes, Chaccour \& Moncunill, 2021; Jin et al., 2021).

102 Concerning COVID-19 immune response, T cell epitopes have the potential to provide long-term

103 protection from SARS-CoV-2. This characteristic allows the detection of memory T cell

104 responses to multiple SARS-CoV-2 proteins, which might contribute to disease control (Chen \&

105 John Wherry, 2020; Karlsson, Humbert \& Buggert, 2020; Sette \& Crotty, 2021).

106 To provide effective T cell epitopes for in vitro peptide-based vaccine design a robust, refined

107 and accurate in silico selection of epitopes is crucial. Despite the many immunoinformatic tools

108 for epitope prediction, the curation for epitope selection is still limited and needs different web

109 servers to complete the analysis. In this paper, we focus on a computational prediction, curation

110 and validation of SARS-CoV-2 epitopes. We have as a central proposal a workflow (EpiCurator)

111 that brings together different approaches for accurate selection epitopes, providing the refined

112 identification of promising SARS-CoV-2 epitopes. To validate the efficacy of this new tool, we

113 use samples of circulating Brazilian lineages available in GISAID (https://www.gisaid.org/) (Elbe

114 \& Buckland-Merrett, 2017) from December 2020 to April 2021.

\section{2. Materials \& Methods}

\section{2.1. Genome retrieval and protein annotation}

117 Genome sequences of 1,652 SARS-CoV-2 genome isolated in Brazil were retrieved from the

118 GISAID database (Elbe \& Buckland-Merrett, 2017) available from December 2020 to April 2021

119 (Table S1). The lineage distribution of the retrieved genomes includes P.1 (Gamma, n=770), P.2

120 (Zeta, $n=525)$, B.1.1.28 $(n=223)$ and B.1.1.33 $(n=136)$. These genomes were analyzed by The

121 Viral Annotation Pipeline and iDentification (VAPiD) (Shean et al., 2019) to determine the

122 amino acid sequence for all SARS-CoV-2 proteins using as reference NC_045512.2 from the

123 NCBI database (https://www.ncbi.nlm.nih.gov/). The final genome processing includes clustering

124 the amino acid sequences for each protein using the CD-HIT package (Li \& Godzik, 2006) with a

125 100\% sequence identity threshold. 
126

127

128

129

130

131

132

133

134

135

136

137

138

139

140

141

142

143

144

145

146

147

148

149

150

151

152

153

154

155

156

157

158

159

160

161

162

163

164

165

166

167

\subsection{Spike protein comparison}

Clustal Omega performed a homology analysis for the Spike protein (Sievers \& Higgins, 2018) and MView tool (Brown, Leroy \& Sander, 1998) among 100 random samples of Brazilian lineages and the VOCs, retrieved from the GISAID database (Elbe \& Buckland-Merrett, 2017), to measure the identity of the sequences to Gamma (P.1) samples.

\subsection{Epitope Prediction}

The T and B cell epitopes prediction was performed using the predictors NetCTL, NetMHCpan, and NetMHCIIpan (https://services.healthtech.dtu.dk/) that allow the high-throughput computing analysis. These predictors support FASTA files containing amino acids marked with " $X$ " in the sequence expanding the possibility of public genomes analysis, even with minor sequencing errors. This prediction features decreasing the pre-processing sequence steps and avoids the falsepositive epitopes provided by joining sequences.

\subsubsection{Prediction of SARS-CoV-2 epitopes.}

To predict CD8+ T cell epitopes, the FASTA sequences of SARS-CoV-2 proteins are processed using NetCTL v1.2 (Larsen et al., 2007). First, the sequences and the HLA class I supertypes, provided by the software, are submitted to select 9-mer peptides (Chen et al., 1994; Sakaguchi et al., 1997; Gfeller et al., 2018), then peptides with amino acids marked with " $X$ ” are removed (Figure 1A). The predicted peptides in NetCTL are further processed using NetMHCpan v.4.0 software (Hoof et al., 2009; Jurtz et al., 2017) to identify epitopes with strong binding affinity to HLA class I alleles. The prediction parameter is based on the predicted percentile rank $\leq 0.5 \%$ and half-maximal inhibitory concentration (IC50) < $500 \mathrm{nM}$ (Chen et al., 1994; Sakaguchi et al., 1997; Gfeller et al., 2018) (Figure 1A). To assess binding affinity, alleles of HLA-A, B, and C loci were selected from Allele Frequency Net Database (Gonzalez-Galarza et al., 2020) by their Brazilian population frequency $>5 \%$ (Table S2).

To predict CD4+ T cell epitopes and estimate binding affinity to HLA class II molecules, the FASTA sequences are processed using NetMHCIIpan v3.2 software (Greenbaum et al., 2011). This tool selects the epitopes with 15-mer peptide lengths based on the predicted percentile rank $\leq 2.0 \%$ and IC50 < $500 \mathrm{nM}$ (Figure 1A). For the affinity prediction, the loci HLA-DRB1, HLADPA1-DPB1, and HLA-DQA1-DQB1 were selected by the phenotypic frequency $>5 \%$ in the Brazilian population (Table S2) from Allele Frequency Net Database (Gonzalez-Galarza et al., 2020). For both cells (CD8+ and CD4+ T cell), the epitopes with broad HLA affinity coverage ( $\geq 3$ alleles) are filtered (Figure $1 \mathrm{~A}$ ).

The prediction of linear B-cell epitopes from SARS-CoV-2 structural proteins was performed by BepiPred v.2.0 software (Jespersen et al., 2017), with a threshold of 0.5 (corresponding specificity $>0.817$ and sensitivity $<0.292$ ) (Figure $1 \mathrm{~A}$ ). Only the epitopes with more than seven and less than 50 amino acid residues in length and sequence without amino acids marked with " $\mathrm{X}$ " are considered for subsequent curation analysis.

\subsection{Accurate selection of epitopes - EpiCurator}

We developed a rigorous analysis workflow for accurate selection of epitopes, the EpiCurator, bringing together a set of filters according to pre-established criteria, optimizing the analysis since it brings tools available to use by high-throughput computing architectures. They also group a series of analyses to guarantee the selection of unpublished and qualified epitopes. The 
168 workflow allows the identification of epitopes by the following analysis: conservancy, homology

169 with the human genome, the overlap between epitopes of different classes, and the identification

170 of epitopes previously published in PubMed Central ${ }^{\circledR}$ (PMC) or available in the IEDB database

171 (Vita et al. 2019) beyond identifying their protein coordinates and mutations (Figure 1B).

172 2.4.1. Prediction of Epitope Conservancy

173 The first module of EpiCurator calculates the conservancy of a predicted epitopes list in SARS-

174 CoV-2 genomes using the BLAST command-line tools (Madden, 2020) (Figure 1B). A

175 customized BLAST database, optimized for shorter sequences (blastp-short task), was generated

176 with proteins of some SARS-CoV-2 circulating lineages from Brazil (B.1.1.28, P.1, P.2)

177 retrieved from the GISAID database (2,787 genome sequences) (Elbe \& Buckland-Merrett,

178 2017). This database allows the comparison among the sequences of the predicted epitopes and

179 the SARS-CoV-2 proteins. Thus, the analysis reports the percentage of identity (conservancy) per

180 epitope using four criteria: $100 \%$ of identity; above $90 \%$; between $70 \%$ and $90 \%$, and less than

$18170 \%$. The epitopes conserved with $100 \%$ of identity in at least $90 \%$ of the genomes are used for

182 further analysis (Figure 1B).

183 2.4.2. Human homology

184 This module uses the human proteins dataset from Ensembl (GRCh38.p13) to identify the

185 predicted epitopes sequence in the human genome. The workflow keeps the human protein

186 sequences in memory to enhance the analysis readiness, searching strictly for the corresponding

187 epitope sequences (exact match). Only the unmatched epitopes are selected, returning a filtered

188 list of epitopes without human homology (Figure 1B).

189 2.4.3. Epitope sequence overlap

190 A comparison is performed between the sequence of the epitopes derived from three groups of

191 prediction (B Cell, HLA Class I, and HLA Class II T Cell). This analysis calculates the

192 intersection and the identity among the lists of epitopes belonging to these groups returning four

193 reports: (i) the intersection of all groups, (ii) B-cell x Class I epitopes, B-Cell x Class II epitopes,

194 and (iii) Class I x Class II epitopes. The result keeps the epitopes with less than 60\% similarity in

195 each report (Figure 1B).

196 2.4.4. Search for epitopes from published articles - EpiMiner

197 The EpiMiner was developed to execute an automatic search of the predicted epitopes list on

198 Pubmed and PMC published papers (Canese \& Weis, 2013) (Figure 1B). It is a pipeline

199 performed in four steps. The first retrieves Pubmed and PMC articles that include the epitopes

200 sequence. The second step extracts the sentences of the body, abstract or tables of the article,

201 highlights the figure captions and breaks apart supplementary table data. The third step executes

202 natural language processing techniques such as tokenization to divide the sentences into words

203 and part-of-speech tagging to filter nouns and verbs (Chowdhury, 2005). These techniques

204 contribute to reducing search time for epitope sequence recognition, eliminating uninformative

205 sentences. The fourth step executes an entity recognition to identify epitopes sequence classified

206 as nouns, saving the sentences and their respective publication into a report. Epitopes information

207 is not searched if it is a part of the pictures or is in supplementary file in text format

208 (i.e. .doc, .docx, .txt, .pdf).

209 2.4.5. IEDB matching 
210 To perform this analysis, the complete ensemble of epitopes from the Immune Epitope Database

211 (IEDB) v3 release (https://www.iedb.org/database_export_v3.php) was retrieved (accessed on

212 July 2021), and the structured files were parsed to filter the epitopes belonging to SARS-CoV-2

213 organisms $(n=1268)$ (Vita et al. 2019). This filtered file is used to calculate the identity between

214 the predicted list of epitopes and the ones retrieved, apprising the IEDB epitope ID and the

215 respective similarity (Figure 1B).

216 2.4.6. Mutation screening

217 Epitopes mutation is reported by comparing the epitope sequence with the SARS-CoV-2 Wuhan

218 protein sequence and assigning the epitopes' coordinates in the respective whole protein. The

219 alignment is conducted with the blastp function of the BLAST command-line tools (Madden,

220 2020). The SARS-CoV-2 reference protein sequences used for alignment are published on the

221 Uniprot Database (https://covid-19.uniprot.org/). This analysis describes the coordinates and

222 presence of mutations in the predicted epitopes (Figure 1B).

223 2.5. Epitope properties

224 2.5.1. Evaluation of antigenicity, toxicity, and immunogenic profile

225 The VaxiJen v2.0 server was applied to analyze the antigenicity of the predicted B cell and T cell

226 epitopes with a conservative score threshold of 0.7 (Doytchinova \& Flower, 2007a,b) (Figure

227 1B). The toxicity is retrieved from the ToxinPred online server with support vector machine

228 (SVM) based methods (threshold -0.4) and e-value cut-off 0.01 (Gupta et al., 2013) (Figure 1B).

229 The allergenic properties are retrieved from the AlgPred2 online server with a hybrid prediction

230 model (threshold 0.5) (Sharma et al., 2020) (Figure 1B). Despite being part of the accurate

231 selection (Figure 1B), they are not available for incorporation into the analytic workflow (item

232 2.4) which prevents us from optimizing their assay. In addition, several on-line servers were used

233 to evaluate the immunogenic profile of the T cell epitopes (Figure 1C). The immunogenicity

234 predictions were performed by the IEDB server (https://www.iedb.org/) (Paul et al. 2015; Calis et

235 al. 2013; Dhanda et al. 2018; Vita et al. 2019). Likewise, their capability to induce interferon-

236 gamma (IFNץ), interleukin-4 (IL-4), interleukin-10 (IL-10), and interleukin-17 (IL-17) (Dhanda,

237 Vir \& Raghava, 2013; Dhanda et al., 2013; Gupta et al., 2017; Nagpal et al., 2017) was evaluated.

238 Additionally, their proinflammatory activity (Gupta et al., 2016) and immunomodulatory

239 potential (Nagpal et al., 2018) was verified. The servers and parameters to evaluate the

240 immunogenic profile are shown in figure 1C.

241 2.5.2. Estimation of population coverage

242 The IEDB's Population Coverage on-line tool (http://tools.iedb.org/population/) is used to

243 analyze how T cell epitopes-HLA binding alleles diverge across ethnicities, regions, and

244 countries around the world (Bui et al. 2006; Vita et al. 2019) (Figure 1C). The predicted epitopes

245 and their respective HLA binding alleles (Table S3) were inputted in the IEDB tool with

246 separated allele class option and a list of countries/regions (Argentina, Brazil, England, France,

247 Italy, Spain, United States, and World) was selected.

248 2.5.3. Docking analysis of the HLA-epitope complex

249 To validate the binding affinity of the predicted epitopes with HLAs structures, docking analysis

250 was performed using the PepDock tool (Lee et al., 2015) of the GALAXY web server (Figure

251 1C). To docking analysis, the Protein Data Bank archive (PDB) of eighth HLA alleles (HLA- 
252 A*01:01, HLA-A*02:01, HLA-B*08:01, HLA-C*12:03, HLA-DRB1*03:01, HLA-

253 DRB1*04:01, HLA-DRB1*12:02 and HLA-DRB1*15:01) was retrieved from the pHLA3D

254 database (Menezes Teles E Oliveira et al., 2019) and RCSB PDB database (Berman et al., 2000;

255 Burley et al., 2021).

256 The PepDock uses the HLA alleles PDBs and the epitopes sequence to perform a template-based

257 model selection and then proceeds to the docking and refinement processes to optimize the

258 energy score ranking ten complex models. The best model of each HLA-epitope complex was

259 selected based on the major similarity score of protein structure and interaction beyond the

260 highest estimated accuracy. Additionally, to identify the free energy and the residue's contacts of

261 the selected complexes, the Prodigy tool (Xue et al., 2016) and Chimera software (Goddard,

262 Huang \& Ferrin, 2005) were used respectively.

\section{2.6. Genomes for EpiCurator pairwise comparison validation}

264 Protein sequences for SARS-CoV-2 isolates reported by (Crooke et al., 2020)(2020(Crooke et al., 265 2020) were identified and retrieved from the Virus Pathogen Resource (ViPR) database ( $\mathrm{n}=$ 266 641,635); additionally, six genome sequences reported by (Kiyotani et al., 2020)(2020(Kiyotani

267 et al., 2020), two sequences for $\mathrm{N}$ and S protein reported by Chen et al., (2020), and five S 268 sequences reported by Chukwudozie et al., (2021) were retrieved from NCBI GenBank. These 269 samples were processed using prediction parameters of the HLA alleles reported by the authors 270 with the approach reported in this Methods section (2.3. and 2.4 analysis). Regarding item 2.4 of

271 Methods, we used only the three main analysis steps (conservancy, human homology and IEDB

272 matching) to compare the selected epitopes ensemble by the papers, and ones chosen

273 independently by our approach, leaving out the EpiMiner analysis since all the epitopes are

274 published.

275 2.7. Multi-epitope construct and structural modelling for EpiCurator validation

276 The RBD epitopes ( $\mathrm{n}=11$ ) were used to construct a multi-epitope sequence connected by

277 specific linkers. The linker aimed to separate the epitopes, so that it improved their expression,

278 folding and stability beyond to prevent their fusion and facilitate the immune processing of

279 antigen (Arai et al., 2001; Kar et al., 2020). In the multi-epitope arrangement are also added an

280 adjuvant (Escherichia coli 50S ribosomal protein L7/L12 (UniProt P0A7K2)) in the N-terminal

281 sequence and a histidine hexamer in the C-terminal portion (Figure 1D, Figure S1).

282 2.7.1. Linear and secondary structure evaluation

283 To evaluate the properties and immune profile of multi-epitope, its linear sequence was submitted

284 to several analyses (Figure 1D) as follow: antigenicity (Doytchinova \& Flower, 2007a; Magnan

285 et al., 2010), allergenicity (Dimitrov et al., 2014b,a), and solubility for cell-free expression

286 analysis (Hebditch et al., 2017), for overexpression analysis (Magnan, Randall \& Baldi, 2009),

287 and structurally solubility profile (Hou et al., 2020). In addition, we assess its physicochemical

288 properties (Gasteiger et al., 2005). The servers and parameters to linear multi-epitope properties

289 evaluation are shown in figure 1D.

290 The linear sequence was also analyzed by the C-IMMSIM server (https://kraken.iac.rm.cnr.it/C-

291 IMMSIM/) to evaluate the in silico immune profile of the multi-epitope construct (Rapin et al.,

292 2010). Two simulations were performed with intervals of 4 or 12 weeks (Saad-Roy et al., 2021;

293 Cobey et al., 2021) (Figure 1D). Furthermore, the secondary structure of the multi-epitope was 
294 evaluated with PSIPRED v.4 with an accuracy of 84.2\% (Jones, 1999; McGuffin, Bryson \&

295 Jones, 2000; Buchan \& Jones, 2019) (Figure 1D)

296 2.7.2. Multi-epitope 3D structure modelling, refinement, and evaluation

297 The tertiary structure multi-epitope construct is modeled by the RaptorX server

298 (http://raptorx.uchicago.edu/ContactMap) that predicts structural properties such as solvent

299 accessibility (ACC) and disorder regions (DIS) (Källberg et al., 2014) (Figure 1D). This tertiary

300 structure was submitted to a refinement process using the GalaxyRefine

301 (http://galaxy.seoklab.org/cgi-bin/submit.cgi?type=REFINE) server which improves global and

302 local model quality by rebuilding all side-chain conformations and applying structural relaxations

303 (Heo, Park \& Seok, 2013). The quality of the refined model is assessed by several parameters, as

304 reported in figure 1D. The refined 3D structure is further evaluated by the ProSA-web server

305 (https://prosa.services.came.sbg.ac.at/prosa.php) to validate structural models checking for

306 potential errors (Berman et al., 2000; Wiederstein \& Sippl, 2007) (Figure 1D). The final refined

307 3D structure was used to perform a docking by the ClusPro server (https://cluspro.bu.edu) (Desta

308 et al., 2020) using the multi-epitope 3D structure and the Toll-Like Receptors TLR4 (PDB: 2Z63)

309 and the TLR3 (PDB: 3CIG). The complex model with the lowest energy is chosen for each

310 receptor (Figure 1D). Their stability is assessed with molecular dynamics simulation on the

311 iMODS server (http://imods.chaconlab.org) that performs Normal Mode Analysis (NMA) to

312 describe functional motions between macromolecules in complexes and simulates feasible

313 trajectories between two conformations (López-Blanco et al., 2014) (Figure 1D).

314 3. Results

315 3.1. Linear epitopes prediction from SARS-CoV-2 genome

316 Three main epitopes prediction comprise HLA Class I (CD8+ T cell), HLA Class II (CD4+ T

317 cell), and B cell. To predict potential CD8+ T cell epitopes, the NetCTL and NetMHCpan,

318 predictive algorithms were performed for all proteins annotated from 1,652 SARS-CoV-2

319 genomes (see Methods). Thus 9-mer epitopes were predicted with strong binding affinity

320 assigned by percentile scores (rank) $\leq 0.5 \%$ across HLA class I alleles frequent in the Brazilian

321 population (Figure 1A). Cumulatively reaching the prediction of 5,261 HLA class I epitopes with

322 HLA promiscuity, binding to $\geq 3$ HLA alleles, implying broad population coverage. The affinity

323 to HLA-C alleles matches the highest epitope binding locus suggesting it could be the best

324 grooves for these epitopes and would lead to the activation of the cell-mediated response (Table

325 1).

326 We also sought to predict potential 15-mer epitopes with binding affinity to HLA class II, using

327 the NetMHCIIpan software (Figure 1A), reaching 7,649 candidate HLA class II epitopes from the

328 SARS-CoV-2 genomes. They have a strong binding affinity to $\geq 3$ HLA alleles across a reference

329 panel of HLA molecules (see Methods). These epitopes showed a preferential affinity for alleles

330 from locus HLA-DRB1 $(n=3,702)$, suggesting that HLA-DRB1 alleles could lead these epitopes

331 to activate the cellular immune response (Table 1).

332 To pairwise the cellular and humoral immune responses activation, the additional prediction of

333 linear B cell epitopes was performed by BepiPred algorithm identifying 257 epitopes from

334 SARS-CoV-2 structural proteins (Table 1).

335 3.2. Quality assessment analysis of the EpiCurator

Peer] reviewing PDF | (2021:06:63076:2:0:NEW 4 Nov 2021) 
336 A robust and refined accurate selection of epitopes is crucial to improve the development of

337 peptide-derived vaccines. To this goal, the EpiCurator brings together six analyses (Conservancy,

338 Human homology, Epitopes overlap, EpiMiner, IEDB matching, and Mutation screening) to the

339 accurately selected epitopes (Figure 1B).

340 To the workflow's quality assessment analysis, we sought to characterize the number of epitopes

341 taken for the main analysis. The epitopes conservancy selected $29.23 \%$ of the predicted epitopes

342 with $100 \%$ identity across at least $90 \%$ of the SARS-CoV-2 samples. Nevertheless, $70.77 \%$ of the

343 predicted epitopes do not correspond to the conservancy parameter (Figure 2, Figure S2). Human

344 homology analysis identified only $0.1 \%$ of the predicted epitopes sequence in the human genome,

345 keeping 99\% accurately selected, unmatched with human sequences (Figure 2, Figure S2). In

346 addition, the screening by the previously published epitopes (EpiMiner and IEDB matching

347 analysis) allowed selected $>80 \%$ of new epitopes, since $17.57 \%$ of them have already been

348 described in the literature and/or IEDB server (Figure 2, Figure S2). To confirm the effectiveness

349 of the EpiMiner we provide all the articles IDs and DOI in which the epitopes were found in table

350 S4.

351 To further assess the quality of the EpiCurator analysis, a pairwise comparison validation was

352 performed. The comparison used NGS data reported by four papers with substantial similarity

353 with our methodologies (Kiyotani et al., 2020; Chen et al., 2020; Crooke et al., 2020;

354 Chukwudozie et al., 2021). The prediction step allows identifying the same HLA class I and HLA

355 class II-restricted T cell epitopes. At the same time, the accurate selection provided by the

356 EpiCurator gathered 51.1\% of the paper's epitopes (Table S5). The conservancy across SARS-

357 CoV-2 genomes samples reached $95,6 \%$ of the paper's epitopes, with only $4.4 \%$ not conserved by

358 our parameters. The human homology step certifies the absence of the article's selected epitopes

359 in the Human genome since the analysis does not recognize the homology of any of them.

360 Interestingly, the IEDB matching step identified $45.6 \%$ of the paper's epitopes as having already

361 been published on the IEDB server showing the importance of this step analysis if we want to

362 describe the epitopes by the first time (Table S5).

363 3.3. Properties of accurately selected epitopes

364 Assembling the workflow analysis results, 199 (3.78\%) HLA class I-restricted T cell epitopes

365 were selected from SARS-CoV-2 proteins, mainly identified in ORF1ab ( $\mathrm{n}=154(77.4 \%)$ ) and

366 Spike glycoprotein $(\mathrm{n}=15(7.5 \%)$ ) (Table 2, Figure 3A). The epitopes keep a high affinity for

367 HLA-C alleles (Figure 3C). The HLA class II epitopes' accurate selection reached 153 (2\%)

368 epitopes, also mainly identified in ORF1ab $(n=111(72.5 \%))$ and Spike glycoprotein $(n=22$

369 (14.4\%)). The HLA class II epitopes showed an affinity prevalence for the HLA-DPA1-DPB1

370 haplotypes (Figure 3D). The comprehensive workflow analysis for B cell epitopes selected

$371 \quad 14.6 \%$ of predicted epitopes with $60 \%$ of the epitopes from the Spike glycoprotein (Table 2).

372 Interestingly, all epitopes have high antigenicity (0.92 \pm 0.30$)$, are non-toxic and non-allergenic

373 (Table S3).

374 Furthermore, the T cell epitopes immunogenic potential was assessed to characterize the

375 capability of inducing in silico protective immune responses. The analysis identified the profile

376 of HLA class I and HLA class II epitopes respectively as follow: IL-4 inducer activity (26.6\% 
377 and 23.8\%), IFNץ production (22.1\% and 39.7\%), immunomodulatory activity (2\% and 3.3\%),

378 and proinflammatory activity (78.9\% and 81.4\%) (Table S3).

379 In addition, to assess the capability to be an in silico Brazilian epitopes candidate, we sought to

380 estimate their distribution among the Brazilian circulating lineages at the time of analysis (P.1,

381 P.2 and B1.1.28). Firstly, we identify the number of epitopes in common across P.1, P.2 and

382 B.1.1.28 lineages identifying the highest proportion of HLA class II epitopes (61.4\%), following

383 by HLA class I (46.7\%) and B cell (14.3\%) (Figure 3B). Thereafter, considering the most

384 representative lineage in Brazil, at the time data was retrieved (P.1). Remarkably, proportions

385 highest than $50 \%$ for all the epitopes were identified, with $70.1 \%$ of HLA class II epitopes,

386 69.8\% of HLA class I, and 51.4\% of B cell epitopes (Figure 3B).

387 In the last analysis, considering the HLA promiscuity epitopes selection, the population coverage

388 for the $\mathrm{T}$ cell epitopes associated with their respective HLA allele binding (Table S3) was

389 estimated by the IEDB server. Notably, the T cell epitopes have a wide population coverage,

390 presenting 99.52\% (HLA class I epitopes) and 100\% (HLA class II epitopes) of cumulative

391 Brazilian population coverage and 98.45\% (HLA class I epitopes) and 99.87\% (HLA class II

392 epitopes) of worldwide population coverage (Table S6).

393 3.4. Epitope-specific RBD Spike as a baseline for validation of EpiCurator selection

394 The EpiCurator allows the selection of the majority of the epitopes in the ORF1ab and Spike

395 glycoprotein. The SARS-CoV-2 Spike glycoprotein has greater prominence concerning the virus

396 and host interaction and has been the main target in epitopes prediction (Shang et al., 2020; Walls

397 et al., 2020). Therefore, we assumed the spike epitopes as a baseline to validate the EpiCurator

398 accurate selection. The Spike epitopes $(n=58)$ were mainly in the $\mathrm{N}$-terminal domain (NTD)

399 (53.4\%) and receptor-binding domain (RBD) (18.9\%) (Figure 4).

400 The RBD epitopes $(n=11)$ comprise three of HLA class I (461-NYNYRYRLF-469, 506-

401 QSYGFQPTY-514, 516-FGYQPYRVV-524), three of HLA class II (322-

402 EKGIYQTSNFRVQPI-336, 322-EKGIYQTSNFRVQPR-336, 443-TGCVIAWNSKNLDSK-

403 457), and five B cell epitopes (332-RVQPTES-338, 424-APGQTGK-430, 424-APGQTGT-430,

404 455-DSKVGGNYN-463, 474-LKPFERD-480) (Figure 4).

405 To evaluate the robust and refined RBD epitopes selection, we perform a diversified analysis.

406 They unveil the highest antigenicity $(1.1 \pm 0.27)$ (Figure 5A) reflecting their ability to bind

407 molecules of adaptive immunity. Notably, their affinity for several HLA alleles are high (0.49 \pm

408 0.15) (Figure 5A) and achieves promiscuity with $\geq 5$ HLA allele binding per epitope (Figure 5B).

409 The RBD epitopes HLA data pairwise with population coverage that remains at around $80 \%$ in

410 the Brazilian and worldwide population (Figure 5B). To further characterize the RBD epitopes,

411 they reach the conservancy of over 99\% across SARS-CoV-2 genomes samples of lineages P.1,

412 P.2 and B.1.1.28 published on GISAID (See Methods) (Table S3).

413 The high conservancy observed suggests that epitope sequences are shared in the circulating

414 Brazilian lineages. Considering the natural homology among the SARS-CoV-2 lineages but

415 regardless of the epitopes' conservancy with other lineages, we expand the identification of the

416 RBD epitopes for diversified samples available in GISAID at the time of writing. Interestingly

417 these epitopes were identified in more than 1 million samples for around 1 thousand lineages of

418 GISAID (Table S7). These findings include some samples of current VOCs: Alpha (B.1.1.7),

Peer] reviewing PDF | (2021:06:63076:2:0:NEW 4 Nov 2021) 
419 Beta (B.1.351), and Delta (B.1.617.2) (Table S7). Concern about the natural lineages’ homology

420

421

422

423

424

425

426

427

428

429

430

431

432

433

434

435

436

437

438

439

440

441

442

443

444

445

446

447

448

449

450

451

452

453

454

455

456

457

458

459

460 mentioned, we perform a comparison among spike glycoprotein of described Brazilian lineages and VOCs. The evaluation of random 100 samples available in GISAID of each lineage shows $99.1 \%$ of identity with $99.9 \%$ of coverage confirming the sequence lineages similarity (Table S8).

One of the mentioned properties of RBD epitopes is the high affinity for HLA alleles. To validate these findings the in silico structural binding performance was assessed by the PepDock docking tool. Thus, the RBD epitopes and the most major HLA alleles in studies with COVID-19 (see Methods) were structurally bonded in complex HLA-epitope (Figure S3). All complexes were seen with high-performance scores demonstrating in silico epitope effectiveness in activating the cell-mediated immune response via MHC presentation. Their high estimated accuracy selected the two most significant HLA-epitope complexes (Table S9, Figure 6). The HLA class I epitope 516-FGYQPYRVV-524 in complex with HLA-A*01:01, HLA-A*02:01, HLA-B*08:01, and HLA-C*12:03; and the HLA class II epitope 443-TGCVIAWNSKNLDSK-457 in complex with HLA-DRB1*03:01 and the HLA-DRB1*12:02 alleles (Table S9, Figure 6). Additionally, the best measured free energy of the complexes was $\Delta \mathrm{G}=-23 \mathrm{kcal} / \mathrm{mol}$ for 461-NYNYRYRLF-469 HLA class I epitope in complex with HLA-A*01:01 and $\Delta \mathrm{G}=-31.6 \mathrm{kcal} / \mathrm{mol}$ for 322-

EKGIYQTSNFRVQPR-336 HLA-class II epitope in complex with the HLA-DRB1*04:01 allele (Table S9, Figure 6). An additional analysis was performed to assess the specific residues involved in the structural binding allowing to identify the specific amino acids in contact between the epitopes and the HLA alleles (Table S9).

\subsection{Multi-epitope construct for in silico validation of RBD epitopes}

To further validate the RBD epitopes and consequently confirm the effectiveness of EpiCurator in providing a curated selection, the epitopes were inserted in a multi-epitope construct. It consists of 287 amino residues, including 11 RBD selected epitopes joined by linkers (Figure S1). The structural appraisal of the secondary structure predicted by the PSIPRED server revealed 31.7\% alpha-helix, 13.5\% beta-strand and the disordered region was 12\% (Figure S4). Some evaluation of the linear sequence of multi-epitope were identified as follows: high antigenicity by the Vaxijen 2.0 (0.87) and the AntigenPro (0.93), good solubility by SolPro (0.91), Protein-sol (0.66), and SOLart (65.80\%), non-allergenic feature by AllerTOP and AllergenFP, hydrophilic property (hydropathicity $=-0.438$ ) and stability (instability index = 20.56), with pI 7.03, and molecular weight calculated to be $29 \mathrm{kDa}$.

Another assessment of linear sequence includes the in silico immunogenic profile provided by the C-IMMSIM immune server. The analysis showed a gradual increase of IgM, IFN- $\gamma$ (associated with both CD8+ T-cell and CD4+ Th1 response as shown in Figure 7A), and IL-2 level after each multi-epitope exposure indicated an elevated immune response (Figure 7B). Besides, an adequate generation of both IgG1 and IgG2 was shown (Figure 7C) with high levels of clonal proliferation of B-cell and T-cell population (Figure S5A and B). In addition, the development of in silico immune memory was assessed by the abundance of different types of B-cells and T-cells (Figure S5C and D).

The favorable in silico immunogenic profile of linear construct led to a multi-epitope 3D modelling to assess TLR binding capability adding validation for the curated selection provided 
461 by the EpiCurator. In this context, we modeled and evaluated the 3D multi-epitope to choose the 462 best model for the TLR binding assay. The best predicted tertiary structure returned by RaptorX 463 has an RMSD score of 11.218 (Figure S6A), 62\% of residues are predicted to be exposed and $46430 \%$ are predicted to be disordered (Figure S6B). The refinement by GalaxyRefine allows the 465 choice of the best model based on its quality scores available in table S10. This model highlights 466 the epitopes according to their type (B cell, HLA class I T cell and HLA class II T cell) (Figure 467 S7). The overall model quality of the refined structure was further validated in the ProSA web 468 (Figure S8A), showing the energy results by position (Figure S8B) with good local quality since 469 all energy values of the residues are negative.

470 Considering the accuracy of the refined model, a docking between our multi-epitope and the 471 immune receptors (TLR3 and TLR4) was performed to check stability and binding affinity. The 472 best complexes (Figure 8 ) for each receptor had free energy values of $\Delta \mathrm{G}=-1048 \mathrm{kcal} / \mathrm{mol}$ 473 (TLR3) and $\Delta \mathrm{G}=-1020 \mathrm{kcal} / \mathrm{mol}$ (TLR4), indicating a high binding affinity. In addition, the 474 stability and physical movements of the complexes were confirmed using molecular dynamics simulation in the iMODS server. The complex results are presented in Figures S9 and S10.

\section{Discussion}

478 Several studies have used immunoinformatic approaches to select B cell and T cell SARS-CoV-2 epitopes for epitopes-based vaccine formulation (Ramana \& Mehla, 2020; Oli et al., 2020; Siracusano, Pastori \& Lopalco, 2020; Yoshida et al., 2021; Ribes, Chaccour \& Moncunill, 2021; Jin et al., 2021). However, current computational methods are limited since they identify a large number of epitopes and need different web servers for accurate selection (Bui et al., 2006; Doytchinova \& Flower, 2007b; Gupta et al., 2013). This study developed an approach that gathered diversified analysis assembled in a workflow (EpiCurator). It accurately selects SARSCoV-2 epitopes with useful in silico properties for an immunogen candidate target. Its effectiveness was validated with a pairwise comparison analysis with four previously published papers (Kiyotani et al., 2020; Chen et al., 2020; Crooke et al., 2020; Chukwudozie et al., 2021). The main analysis of this validation was the IEDB matching that promotes a robust and refined selection of epitopes which confirms the importance of the IEDB database in the epitopes analysis studies (Beaver, Bourne \& Ponomarenko, 2007). In addition, our approach can be used for extensive scale search and high-throughput computing analysis with the SARS-CoV2 genomes available, an advantage to analyze a pandemic emergent virus (Ojha et al., 2020; Minervina et al., 2021; Pham et al., 2021).

494 Remarkably, our workflow conducts a thorough and facilitated accurate selection that enables us to prioritize the epitopes candidates. The EpiCurator recognizes patterns of cross-conservation with SARS-CoV-2 and human epitopes, eliminating the ones with significant human homology (Meyers et al., 2021). In addition, notably identifying epitopes in published articles, ensuring the selection in silico candidates with a plausible assumption to be described by the first time in this study. Consequently, this analysis might also validate the effectiveness of our workflow's

501 accurate selection analysis, since our epitopes were identified in different studies previously

502 published (Prachar et al., 2020). The workflow also allows the selection of epitopes by high conservancy ( $\geq 90 \%$ ), across more than two thousand SARS-CoV-2 circulating Brazilian lineages 
503 in accordance with previous reports for SARS-CoV-2 epitopes selection (Zaheer et al., 2020;

504 Mahapatra et al., 2020; Mallajosyula et al., 2021).

505 Three main phases comprise our approach to SARS-CoV-2 epitopes identification, the prediction,

506 accurate selection and epitopes validation. Therefore, taking the spike glycoprotein epitopes as

507 parameters, they represent $10 \%$ of the predicted epitopes and $2 \%$ of the accurately selected

508 similarly to those presented in other reports (Kiyotani et al., 2020; Crooke et al., 2020) with the

509 resembling result for the other proteins. Indeed, the spike data give us an advantage since they

510 were used to optimize the validation of the epitopes accurately selected by EpiCurator. Thus, they

511 are taken as a baseline to conduct a thorough analysis. This baseline is reached since the Spike

512 plays the most crucial role in the entry of viral particles into host cells, promoting an effective

513 infection (Ou et al., 2020). These characteristics make the spike a chosen target for epitope

514 screening leading to vaccine development (Shang et al., 2020; Walls et al., 2020; Lin et al.,

515 2020). Pertaining to the Spike, the RBD region induces responses that block S protein binding

516 with the human cell receptor, neutralizing SARS-CoV-2 infection (Rakib et al., 2020; Yang et al.,

517 2020), having substantial importance to peptide-based vaccine studies. Considering this

518 importance, we prioritize 11 epitopes of this study identified on Spike RBD regions.

519 The RBD epitopes were identified in several SARS-CoV-2 lineages emphasizing samples of the

520 current VOCs. They have favorable in silico structure interactions with the main HLA alleles

521 related to the COVID-19 response (Tavasolian et al., 2020; Tomita et al., 2020; Shkurnikov et al.,

522 2021). Thereby, it is plausible to assume that these epitopes could be responsible for the strong

523 activation of the cell-mediated immune response (Patronov et al., 2011; Sarma, Olotu \& Soliman,

524 2021) in case of an experimental assay. In addition, the RBD epitopes have a broad populational

525 coverage consistent with studies for SARS-CoV-2 epitopes identified in lineages isolated from

526 India, England and the United States (Mallavarpu Ambrose et al., 2021). These findings

527 combined with the high conservancy, antigenicity and immunogenicity suggest these epitopes' in

528 silico immunogen profile (Zheng \& Song, 2020; Mallavarpu Ambrose et al., 2021; Jahangirian et

529 al., 2021), validating the accurate selection of EpiCurator.

530 Indeed, the RBD epitopes have substantial evidence and are seen as good epitope candidates.

531 Despite sharing this evidence, they could together increase the immune responses and confer

532 better protection against SARS-CoV-2 (Jahangirian et al., 2021). Accepting this assumption, they

533 were used to design a multi-epitope construct to further validate their immunogenic properties

534 (Kalita et al., 2020; Mohammad et al., 2020; Singh et al., 2020; Yang, Bogdan \& Nazarian, 2021;

535 Lim et al., 2021; Sharma et al., 2021). The multi-epitope has the capability of in silico activation

536 of memory B and T-cell with Th1 response. Several experimental studies about immune response

537 against COVID-19 endorse these findings (Lipsitch et al., 2020; Hartley et al., 2020; Ghazavi et

538 al., 2021; Quast \& Tarlinton, 2021; Tarke et al., 2021). On the other hand, no response Th17 was

539 found in the in silico assay, a common response that characterizes the severe COVID-19 profile

540 (Wu \& Yang, 2020). Additionally, the multi-epitope had low binding energy and high stability of

541 in silico structure interactions with TLR3 and TLR4 similar to other computational studies (Kar

542 et al., 2020; Rahman et al., 2020; Yang, Bogdan \& Nazarian, 2021; Nemati et al., 2021; Saba et

543 al., 2021). This interaction is important since TLR is an innate immune receptor that recognizes

544 viral proteins and triggers infection resistance (O’Neill, Golenbock \& Bowie, 2013). 
545 Furthermore, TLR3 and TLR4 are specifically related by different studies as part of immune 546 response in COVID-19 (Patra, Chandra Das \& Mukherjee, 2021; Khanmohammadi \& Rezaei, 547 2021; Kaushik, Bhandari \& Kuhad, 2021). The findings in multi-epitope analysis confirm the

548 importance of the RBD epitopes, selected by the EpiCurator, as good in silico immunogens

549 candidates, reinforcing our approach's efficiency for a curated selection of epitopes.

550

\section{Conclusions}

552

It is important to reinforce that this work focuses on a computational prediction and selection of

553 epitopes seeking to perform several in silico validation. Therefore, our approach is useful to researchers designing an experimental peptide-based vaccine to control the disease. Since, indeed, the development of an effective vaccine requires a detailed experimental investigation of the immunological correlations with SARS-CoV-2. Regarding the computational analysis, assembling the accurate selection of epitopes and their validation consolidate our approach as a helpful workflow analysis to provide epitopes for immunogens. These epitopes could significantly activate the in silico immune response against some circulating Brazilian variants. Furthermore, considering that the pandemic is still ongoing, our approach could contribute to continuous monitoring and identification of new SARS-CoV-2 epitopes according to the emergence of variants over time.

563

\section{Acknowledgements}

565

We would like to thank all the authors and the administrators of the GISAID and IEDB databases, allowing this study to be properly conducted.

568 The authors acknowledge the National Laboratory for Scientific Computing (LNCC/MCTI, Brazil) for providing HPC resources of the SDumont supercomputer, which has contributed to the results of the research reported within this paper. URL: http://sdumont.lncc.br

571

572

573

574

575

\section{References}

Andreano E, Piccini G, Licastro D, Casalino L, Johnson NV, Paciello I, Dal Monego S, Pantano E, Manganaro N, Manenti A, Manna R, Casa E, Hyseni I, Benincasa L, Montomoli E, Amaro RE, McLellan JS, Rappuoli R. 2021. SARS-CoV-2 escape from a highly neutralizing COVID-19 convalescent plasma. Proceedings of the National Academy of Sciences of the United States of America 118. DOI: 10.1073/pnas.2103154118.

Arai R, Ueda H, Kitayama A, Kamiya N, Nagamune T. 2001. Design of the linkers which effectively separate domains of a bifunctional fusion protein. Protein engineering 14:529532. DOI: 10.1093/protein/14.8.529.

Badiani AA, Patel JA, Ziolkowski K, Nielsen FBH. 2020. Pfizer: The miracle vaccine for COVID-19? Public health in practice (Oxford, England) 1:100061. DOI: 10.1016/j.puhip.2020.100061.

Bashir Z, Ahmad SU, Kiani BH, Jan Z, Khan N, Khan U, Haq I, Zahir F, Qadus A, Mahmood T. 
588

589

590

591

592

593

594

595

596

597

598

599

600

601

602

603

604

605

606

607

608

609

610

611

612

613

614

615

616

617

618

619

620

621

622

623

624

625

626

627

628

629

630

631

632

633

634

635

636
2021. Immunoinformatics approaches to explore $B$ and $T$ cell epitope-based vaccine designing for SARS-CoV-2 Virus. Pakistan journal of pharmaceutical sciences 34:345-352. DOI: 10.36721/pjps.2021.34.1.sup.345-352.1.

Beaver JE, Bourne PE, Ponomarenko JV. 2007. EpitopeViewer: a Java application for the visualization and analysis of immune epitopes in the Immune Epitope Database and Analysis Resource (IEDB). Immunome research 3:3. DOI: 10.1186/1745-7580-3-3.

Berman HM, Westbrook J, Feng Z, Gilliland G, Bhat TN, Weissig H, Shindyalov IN, Bourne PE. 2000. The Protein Data Bank. Nucleic acids research 28:235-242. DOI: 10.1093/nar/28.1.235.

Bloom DE, Cadarette D, Ferranna M, Hyer RN, Tortorice DL. 2021. How New Models Of Vaccine Development For COVID-19 Have Helped Address An Epic Public Health Crisis. Health affairs 40:410-418. DOI: 10.1377/hlthaff.2020.02012.

Brown NP, Leroy C, Sander C. 1998. MView: a web-compatible database search or multiple alignment viewer. Bioinformatics 14:380-381. DOI: 10.1093/bioinformatics/14.4.380.

Brüssow H. 2021. COVID-19: emergence and mutational diversification of SARS-CoV-2. Microbial biotechnology 14:756-768. DOI: 10.1111/1751-7915.13800.

Buchan DWA, Jones DT. 2019. The PSIPRED Protein Analysis Workbench: 20 years on. Nucleic acids research 47:W402-W407. DOI: 10.1093/nar/gkz297.

Bui H-H, Sidney J, Dinh K, Southwood S, Newman MJ, Sette A. 2006. Predicting population coverage of T-cell epitope-based diagnostics and vaccines. BMC bioinformatics 7:153. DOI: 10.1186/1471-2105-7-153.

Bulla HAM. 2021. COVID -19: EFFICACY AND SAFETY PROFILE OF MAIN VACCINES APPROVED FOR EMERGENCY USE AUTHORIZATION IN 2021. International Journal of Research -GRANTHAALAYAH 9:271-283. DOI: 10.29121/granthaalayah.v9.i7.2021.4062.

Burley SK, Bhikadiya C, Bi C, Bittrich S, Chen L, Crichlow GV, Christie CH, Dalenberg K, Di Costanzo L, Duarte JM, Dutta S, Feng Z, Ganesan S, Goodsell DS, Ghosh S, Green RK, Guranović V, Guzenko D, Hudson BP, Lawson CL, Liang Y, Lowe R, Namkoong H, Peisach E, Persikova I, Randle C, Rose A, Rose Y, Sali A, Segura J, Sekharan M, Shao C, Tao Y-P, Voigt M, Westbrook JD, Young JY, Zardecki C, Zhuravleva M. 2021. RCSB Protein Data Bank: powerful new tools for exploring 3D structures of biological macromolecules for basic and applied research and education in fundamental biology, biomedicine, biotechnology, bioengineering and energy sciences. Nucleic acids research 49:D437-D451. DOI: 10.1093/nar/gkaa1038.

Calis JJA, Maybeno M, Greenbaum JA, Weiskopf D, De Silva AD, Sette A, Keşmir C, Peters B. 2013. Properties of MHC class I presented peptides that enhance immunogenicity. PLoS computational biology 9:e1003266. DOI: 10.1371/journal.pcbi.1003266.

Canese K, Weis S. 2013. Pubmed: the bibliographic database. In: The NCBI Handbook [Internet]. National Center for Biotechnology Information (US),.

Cella E, Benedetti F, Fabris S, Borsetti A, Pezzuto A, Ciotti M, Pascarella S, Ceccarelli G, Zella D, Ciccozzi M, Giovanetti M. 2021. SARS-CoV-2 Lineages and Sub-Lineages Circulating Worldwide: A Dynamic Overview. Chemotherapy:1-5. DOI: 10.1159/000515340.

Chakraborty C, Sharma AR, Bhattacharya M, Sharma G, Lee S-S. 2020. The 2019 novel coronavirus disease (COVID-19) pandemic: A zoonotic prospective. Asian Pacific journal of tropical medicine 13:242. DOI: 10.4103/1995-7645.281613.

Chen Z, John Wherry E. 2020. T cell responses in patients with COVID-19. Nature reviews. Immunology 20:529-536. DOI: 10.1038/s41577-020-0402-6.

Chen Y, Sidney J, Southwood S, Cox AL, Sakaguchi K, Henderson RA, Appella E, Hunt DF, Sette A, Engelhard VH. 1994. Naturally processed peptides longer than nine amino acid 
637

638

639

640

641

642

643

644

645

646

647

648

649

650

651

652

653

654

655

656

657

658

659

660

661

662

663

664

665

666

667

668

669

670

671

672

673

674

675

676

677

678

679

680

681

682

683

684

685 residues bind to the class I MHC molecule HLA-A2.1 with high affinity and in different conformations. Journal of immunology 152:2874-2881.

Chen H-Z, Tang L-L, Yu X-L, Zhou J, Chang Y-F, Wu X. 2020. Bioinformatics analysis of epitope-based vaccine design against the novel SARS-CoV-2. Infectious diseases of poverty 9:88. DOI: 10.1186/s40249-020-00713-3.

Chowdhury GG. 2005. Natural language processing. Annual review of information science and technology 37:51-89. DOI: 10.1002/aris.1440370103.

Chukwudozie OS, Gray CM, Fagbayi TA, Chukwuanukwu RC, Oyebanji VO, Bankole TT, Adewole RA, Daniel EM. 2021. Immuno-informatics design of a multimeric epitope peptide based vaccine targeting SARS-CoV-2 spike glycoprotein. PloS one 16:e0248061. DOI: 10.1371/journal.pone.0248061.

Cobey S, Larremore DB, Grad YH, Lipsitch M. 2021. Concerns about SARS-CoV-2 evolution should not hold back efforts to expand vaccination. Nature reviews. Immunology 21:330 335. DOI: 10.1038/s41577-021-00544-9.

Cohen J, Normile D. 2020. New SARS-like virus in China triggers alarm. Science 367:234-235. DOI: 10.1126/science.367.6475.234.

Crooke SN, Ovsyannikova IG, Kennedy RB, Poland GA. 2020. Immunoinformatic identification of B cell and T cell epitopes in the SARS-CoV-2 proteome. Scientific reports 10:14179. DOI: 10.1038/s41598-020-70864-8.

Desta IT, Porter KA, Xia B, Kozakov D, Vajda S. 2020. Performance and Its Limits in Rigid Body Protein-Protein Docking. Structure 28:1071-1081.e3. DOI: 10.1016/j.str.2020.06.006.

Dhanda SK, Gupta S, Vir P, Raghava GPS. 2013. Prediction of IL4 inducing peptides. Clinical \& developmental immunology 2013:263952. DOI: 10.1155/2013/263952.

Dhanda SK, Karosiene E, Edwards L, Grifoni A, Paul S, Andreatta M, Weiskopf D, Sidney J, Nielsen M, Peters B, Sette A. 2018. Predicting HLA CD4 Immunogenicity in Human Populations. Frontiers in immunology 9:1369. DOI: 10.3389/fimmu.2018.01369.

Dhanda SK, Vir P, Raghava GPS. 2013. Designing of interferon-gamma inducing MHC class-II binders. Biology direct 8:30. DOI: 10.1186/1745-6150-8-30.

Dimitrov I, Bangov I, Flower DR, Doytchinova I. 2014a. AllerTOP v.2--a server for in silico prediction of allergens. Journal of molecular modeling 20:2278. DOI: 10.1007/s00894-0142278-5.

Dimitrov I, Naneva L, Doytchinova I, Bangov I. 2014b. AllergenFP: allergenicity prediction by descriptor fingerprints. Bioinformatics 30:846-851. DOI: 10.1093/bioinformatics/btt619.

Di Natale C, La Manna S, De Benedictis I, Brandi P, Marasco D. 2020. Perspectives in PeptideBased Vaccination Strategies for Syndrome Coronavirus 2 Pandemic. Frontiers in pharmacology 11:578382. DOI: 10.3389/fphar.2020.578382.

Doytchinova IA, Flower DR. 2007a. VaxiJen: a server for prediction of protective antigens, tumour antigens and subunit vaccines. BMC bioinformatics 8:4. DOI: 10.1186/1471-2105-84.

Doytchinova IA, Flower DR. 2007b. Identifying candidate subunit vaccines using an alignmentindependent method based on principal amino acid properties. Vaccine 25:856-866. DOI: 10.1016/j.vaccine.2006.09.032.

Elbe S, Buckland-Merrett G. 2017. Data, disease and diplomacy: GISAID’s innovative contribution to global health. Global challenges (Hoboken, $N J$ ) 1:33-46. DOI: 10.1002/gch2.1018.

Faria NR, Claro IM, Candido D, Franco LAM, Andrade PS, Coletti TM, Silva CAM, Sales FC, Manuli ER, Aguiar RS, Gaburo N, Camilo C da C, Fraiji NA, Crispim MAE, Carvalho M do PSS, Rambaut A, Loman N, Pybus OG, Sabino EC. 2020.Genomic characterisation of an emergent SARS-CoV-2 lineage in Manaus: preliminary findings. Available at 
686

687

688

689

690

691

692

693

694

695

696

697

698

699

700

701

702

703

704

705

706

707

708

709

710

711

712

713

714

715

716

717

718

719

720

721

722

723

724

725

726

727

728

729

730

731

732

733

734 https://virological.org/t/genomic-characterisation-of-an-emergent-sars-cov-2-lineage-inmanaus-preliminary-findings/586 (accessed June 2021).

Fast E, Altman RB, Chen B. 2020. Potential T-cell and B-cell Epitopes of 2019-nCoV. bioRxiv:2020.02.19.955484. DOI: 10.1101/2020.02.19.955484.

Fatoba AJ, Maharaj L, Adeleke VT, Okpeku M, Adeniyi AA, Adeleke MA. 2021. Immunoinformatics prediction of overlapping CD8+ T-cell, IFN- $\gamma$ and IL-4 inducer CD4+ T-cell and linear B-cell epitopes based vaccines against COVID-19 (SARS-CoV-2). Vaccine 39:1111-1121. DOI: 10.1016/j.vaccine.2021.01.003.

Gao Q, Bao L, Mao H, Wang L, Xu K, Yang M, Li Y, Zhu L, Wang N, Lv Z, Gao H, Ge X, Kan B, Hu Y, Liu J, Cai F, Jiang D, Yin Y, Qin C, Li J, Gong X, Lou X, Shi W, Wu D, Zhang H, Zhu L, Deng W, Li Y, Lu J, Li C, Wang X, Yin W, Zhang Y, Qin C. 2020. Development of an inactivated vaccine candidate for SARS-CoV-2. Science 369:77-81. DOI: 10.1126/science.abc1932.

Gasteiger E, Hoogland C, Gattiker A, Duvaud S, Wilkins MR, Appel RD, Amos B. 2005. Protein Identification and Analysis Tools on the ExPASy Server. In: Walker JM ed. The Proteomics Protocols Handbook. Humana Press, 571-607.

Geers D, Shamier MC, Bogers S, den Hartog G, Gommers L, Nieuwkoop NN, Schmitz KS, Rijsbergen LC, van Osch JAT, Dijkhuizen E, Smits G, Comvalius A, van Mourik D, Caniels TG, van Gils MJ, Sanders RW, Oude Munnink BB, Molenkamp R, de Jager HJ, Haagmans BL, de Swart RL, Koopmans MPG, van Binnendijk RS, de Vries RD, GeurtsvanKessel CH. 2021. SARS-CoV-2 variants of concern partially escape humoral but not T-cell responses in COVID-19 convalescent donors and vaccinees. Science immunology 6. DOI: 10.1126/sciimmunol.abj1750.

Gfeller D, Guillaume P, Michaux J, Pak H-S, Daniel RT, Racle J, Coukos G, Bassani-Sternberg M. 2018. The Length Distribution and Multiple Specificity of Naturally Presented HLA-I Ligands. Journal of immunology 201:3705-3716. DOI: 10.4049/jimmunol.1800914.

Ghazavi A, Ganji A, Keshavarzian N, Rabiemajd S, Mosayebi G. 2021. Cytokine profile and disease severity in patients with COVID-19. Cytokine 137:155323. DOI: 10.1016/j.cyto.2020.155323.

Goddard TD, Huang CC, Ferrin TE. 2005. Software extensions to UCSF chimera for interactive visualization of large molecular assemblies. Structure 13:473-482. DOI: 10.1016/j.str.2005.01.006.

Gonzalez-Galarza FF, McCabe A, Santos EJMD, Jones J, Takeshita L, Ortega-Rivera ND, CidPavon GMD, Ramsbottom K, Ghattaoraya G, Alfirevic A, Middleton D, Jones AR. 2020. Allele frequency net database (AFND) 2020 update: gold-standard data classification, open access genotype data and new query tools. Nucleic acids research 48:D783-D788. DOI: 10.1093/nar/gkz1029.

Greaney AJ, Loes AN, Crawford KHD, Starr TN, Malone KD, Chu HY, Bloom JD. 2021. Comprehensive mapping of mutations in the SARS-CoV-2 receptor-binding domain that affect recognition by polyclonal human plasma antibodies. Cell host \& microbe 29:463476.e6. DOI: 10.1016/j.chom.2021.02.003.

Greenbaum J, Sidney J, Chung J, Brander C, Peters B, Sette A. 2011. Functional classification of class II human leukocyte antigen (HLA) molecules reveals seven different supertypes and a surprising degree of repertoire sharing across supertypes. Immunogenetics 63:325-335. DOI: 10.1007/s00251-011-0513-0.

Gupta S, Kapoor P, Chaudhary K, Gautam A, Kumar R, Open Source Drug Discovery Consortium, Raghava GPS. 2013. In silico approach for predicting toxicity of peptides and proteins. PloS one 8:e73957. DOI: 10.1371/journal.pone.0073957.

Gupta S, Madhu MK, Sharma AK, Sharma VK. 2016. ProInflam: a webserver for the prediction 
735

736

737

738

739

740

741

742

743

744

745

746

747

748

749

750

751

752

753

754

755

756

757

758

759

760

761

762

763

764

765

766

767

768

769

770

771

772

773

774

775

776

777

778

779

780

781

782

783 of proinflammatory antigenicity of peptides and proteins. Journal of translational medicine 14:1-10. DOI: 10.1186/s12967-016-0928-3.

Gupta S, Mittal P, Madhu MK, Sharma VK. 2017. IL17eScan: A Tool for the Identification of Peptides Inducing IL-17 Response. Frontiers in immunology 8:1430. DOI:

10.3389/fimmu.2017.01430.

Hartley GE, Edwards ESJ, Aui PM, Varese N, Stojanovic S, McMahon J, Peleg AY, Boo I, Drummer HE, Hogarth PM, O’Hehir RE, van Zelm MC. 2020. Rapid generation of durable B cell memory to SARS-CoV-2 spike and nucleocapsid proteins in COVID-19 and convalescence. Science immunology 5. DOI: 10.1126/sciimmunol.abf8891.

Hebditch M, Carballo-Amador MA, Charonis S, Curtis R, Warwicker J. 2017. Protein-Sol: a web tool for predicting protein solubility from sequence. Bioinformatics 33:3098-3100. DOI: 10.1093/bioinformatics/btx345.

Heo L, Park H, Seok C. 2013. GalaxyRefine: Protein structure refinement driven by side-chain repacking. Nucleic acids research 41:W384-8. DOI: 10.1093/nar/gkt458.

Hoffmann M, Arora P, Groß R, Seidel A, Hörnich BF, Hahn AS, Krüger N, Graichen L, Hofmann-Winkler H, Kempf A, Winkler MS, Schulz S, Jäck H-M, Jahrsdörfer B, Schrezenmeier H, Müller M, Kleger A, Münch J, Pöhlmann S. 2021. SARS-CoV-2 variants B.1.351 and P.1 escape from neutralizing antibodies. Cell 184:2384-2393.e12. DOI: 10.1016/j.cell.2021.03.036.

Hoof I, Peters B, Sidney J, Pedersen LE, Sette A, Lund O, Buus S, Nielsen M. 2009. NetMHCpan, a method for MHC class I binding prediction beyond humans. Immunogenetics 61:1-13. DOI: 10.1007/s00251-008-0341-z.

Hou Q, Kwasigroch JM, Rooman M, Pucci F. 2020. SOLart: a structure-based method to predict protein solubility and aggregation. Bioinformatics 36:1445-1452. DOI: 10.1093/bioinformatics/btz773.

Huang C, Wang Y, Li X, Ren L, Zhao J, Hu Y, Zhang L, Fan G, Xu J, Gu X, Cheng Z, Yu T, Xia J, Wei Y, Wu W, Xie X, Yin W, Li H, Liu M, Xiao Y, Gao H, Guo L, Xie J, Wang G, Jiang R, Gao Z, Jin Q, Wang J, Cao B. 2020. Clinical features of patients infected with 2019 novel coronavirus in Wuhan, China. The Lancet 395:497-506. DOI: 10.1016/S01406736(20)30183-5.

Hudu SA, Shinkafi SH, Umar S. 2016. AN OVERVIEW OF RECOMBINANT VACCINE TECHNOLOGY, ADJUVANTS AND VACCINE DELIVERY METHODS. International journal of pharmacy and pharmaceutical sciences:19-24. DOI: 10.22159/ijpps.2016v8i11.14311.

Jahangirian E, Jamal GA, Nouroozi M, Mohammadpour A. 2021. A reverse vaccinology and immunoinformatics approach for designing a multiepitope vaccine against SARS-CoV-2. Immunogenetics. DOI: 10.1007/s00251-021-01228-3.

Jespersen MC, Peters B, Nielsen M, Marcatili P. 2017. BepiPred-2.0: improving sequence-based B-cell epitope prediction using conformational epitopes. Nucleic acids research 45:W24W29. DOI: 10.1093/nar/gkx346.

Jin X, Ding Y, Sun S, Wang X, Zhou Z, Liu X, Li M, Chen X, Shen A, Wu Y, Liu B, Zhang J, Li J, Yang Y, Qiu H, Shen C, He Y, Zhao G. 2021. Screening of HLA-A restricted T cell epitopes of SARS-CoV-2 and induction of CD8+ T cell responses in HLA-A transgenic mice. bioRxiv:2021.04.01.438020. DOI: 10.1101/2021.04.01.438020.

Jones DT. 1999. Protein secondary structure prediction based on position-specific scoring matrices. Journal of molecular biology 292:195-202. DOI: 10.1006/jmbi.1999.3091.

Jurtz V, Paul S, Andreatta M, Marcatili P, Peters B, Nielsen M. 2017. NetMHCpan-4.0: Improved Peptide-MHC Class I Interaction Predictions Integrating Eluted Ligand and Peptide Binding Affinity Data. Journal of immunology 199:3360-3368. DOI: 
784

785

786

787

788

789

790

791

792

793

794

795

796

797

798

799

800

801

802

803

804

805

806

807

808

809

810

811

812

813

814

815

816

817

818

819

820

821

822

823

824

825

826

827

828

829

830

831

832 10.4049/jimmunol.1700893.

Kalita P, Padhi AK, Zhang KYJ, Tripathi T. 2020. Design of a peptide-based subunit vaccine against novel coronavirus SARS-CoV-2. Microbial pathogenesis 145:104236. DOI: 10.1016/j.micpath.2020.104236.

Källberg M, Margaryan G, Wang S, Ma J, Xu J. 2014. RaptorX server: a resource for templatebased protein structure modeling. Methods in molecular biology 1137:17-27. DOI: 10.1007/978-1-4939-0366-5_2.

Karlsson AC, Humbert M, Buggert M. 2020. The known unknowns of T cell immunity to COVID-19. Science immunology 5. DOI: 10.1126/sciimmunol.abe8063.

Kar T, Narsaria U, Basak S, Deb D, Castiglione F, Mueller DM, Srivastava AP. 2020. A candidate multi-epitope vaccine against SARS-CoV-2. Scientific reports 10:10895. DOI: 10.1038/s41598-020-67749-1.

Kaushik D, Bhandari R, Kuhad A. 2021. TLR4 as a therapeutic target for respiratory and neurological complications of SARS-CoV-2. Expert opinion on therapeutic targets 25:491508. DOI: 10.1080/14728222.2021.1918103.

Kazi A, Chuah C, Majeed ABA, Leow CH, Lim BH, Leow CY. 2018. Current progress of immunoinformatics approach harnessed for cellular- and antibody-dependent vaccine design. Pathogens and global health 112:123-131. DOI: 10.1080/20477724.2018.1446773.

Khanmohammadi S, Rezaei N. 2021. Role of Toll-like receptors in the pathogenesis of COVID19. Journal of medical virology 93:2735-2739. DOI: 10.1002/jmv.26826.

Kiyotani K, Toyoshima Y, Nemoto K, Nakamura Y. 2020. Bioinformatic prediction of potential T cell epitopes for SARS-Cov-2. Journal of human genetics 65:569-575. DOI: 10.1038/s10038-020-0771-5.

Knoll MD, Wonodi C. 2021. Oxford-AstraZeneca COVID-19 vaccine efficacy. The Lancet 397:72-74. DOI: 10.1016/S0140-6736(20)32623-4.

Krammer F. 2020. SARS-CoV-2 vaccines in development. Nature 586:516-527. DOI: 10.1038/s41586-020-2798-3.

Kyriakidis NC, López-Cortés A, González EV, Grimaldos AB, Prado EO. 2021. SARS-CoV-2 vaccines strategies: a comprehensive review of phase 3 candidates. NPJ vaccines 6:28. DOI: 10.1038/s41541-021-00292-w.

Larsen MV, Lundegaard C, Lamberth K, Buus S, Lund O, Nielsen M. 2007. Large-scale validation of methods for cytotoxic T-lymphocyte epitope prediction. BMC bioinformatics 8:424. DOI: 10.1186/1471-2105-8-424.

Lee H, Heo L, Lee MS, Seok C. 2015. GalaxyPepDock: a protein-peptide docking tool based on interaction similarity and energy optimization. Nucleic acids research 43:W431-5. DOI: 10.1093/nar/gkv495.

Li W, Godzik A. 2006. Cd-hit: a fast program for clustering and comparing large sets of protein or nucleotide sequences. Bioinformatics 22:1658-1659. DOI: 10.1093/bioinformatics/btl158.

Lim HX, Lim J, Jazayeri SD, Poppema S, Poh CL. 2021. Development of multi-epitope peptidebased vaccines against SARS-CoV-2. Biomedical journal 44:18-30. DOI: 10.1016/j.bj.2020.09.005.

Lin L, Ting S, Yufei H, Wendong L, Yubo F, Jing Z. 2020. Epitope-based peptide vaccines predicted against novel coronavirus disease caused by SARS-CoV-2. Virus research 288:198082. DOI: 10.1016/j.virusres.2020.198082.

Lipsitch M, Grad YH, Sette A, Crotty S. 2020. Cross-reactive memory T cells and herd immunity to SARS-CoV-2. Nature reviews. Immunology 20:709-713. DOI: 10.1038/s41577-02000460-4.

Liu Z, VanBlargan LA, Bloyet L-M, Rothlauf PW, Chen RE, Stumpf S, Zhao H, Errico JM, 
833

834

835

836

837

838

839

840

841

842

843

844

845

846

847

848

849

850

851

852

853

854

855

856

857

858

859

860

861

862

863

864

865

866

867

868

869

870

871

872

873

874

875

876

877

878

879

880

881
Theel ES, Liebeskind MJ, Alford B, Buchser WJ, Ellebedy AH, Fremont DH, Diamond MS, Whelan SPJ. 2021. Identification of SARS-CoV-2 spike mutations that attenuate monoclonal and serum antibody neutralization. Cell host \& microbe 29:477-488.e4. DOI:

10.1016/j.chom.2021.01.014.

Livingston EH, Malani PN, Creech CB. 2021. The Johnson \& Johnson Vaccine for COVID-19. JAMA: the journal of the American Medical Association 325:1575. DOI:

10.1001/jama.2021.2927.

López-Blanco JR, Aliaga JI, Quintana-Ortí ES, Chacón P. 2014. iMODS: internal coordinates normal mode analysis server. Nucleic acids research 42:W271-6. DOI: 10.1093/nar/gku339.

Lu G, Shan S, Zainab B, Ayaz Z, He J, Xie Z, Rashid U, Zhang D, Mehmood Abbasi A. 2021. Novel vaccine design based on genomics data analysis: A review. Scandinavian journal of immunology 93:e12986. DOI: 10.1111/sji.12986.

Madden T. 2020. User Manual. National Center for Biotechnology Information (US).

Magnan CN, Randall A, Baldi P. 2009. SOLpro: accurate sequence-based prediction of protein solubility. Bioinformatics 25:2200-2207. DOI: 10.1093/bioinformatics/btp386.

Magnan CN, Zeller M, Kayala MA, Vigil A, Randall A, Felgner PL, Baldi P. 2010. Highthroughput prediction of protein antigenicity using protein microarray data. Bioinformatics 26:2936-2943. DOI: 10.1093/bioinformatics/btq551.

Mahapatra SR, Sahoo S, Dehury B, Raina V, Patro S, Misra N, Suar M. 2020. Designing an efficient multi-epitope vaccine displaying interactions with diverse HLA molecules for an efficient humoral and cellular immune response to prevent COVID-19 infection. Expert review of vaccines 19:871-885. DOI: 10.1080/14760584.2020.1811091.

Mahase E. 2020. Covid-19: Moderna vaccine is nearly 95\% effective, trial involving high risk and elderly people shows. BMJ 371. DOI: 10.1136/bmj.m4471.

Mallajosyula V, Ganjavi C, Chakraborty S, McSween AM, Pavlovitch-Bedzyk AJ, Wilhelmy J, Nau A, Manohar M, Nadeau KC, Davis MM. 2021. CD8+ T cells specific for conserved coronavirus epitopes correlate with milder disease in COVID-19 patients. Science immunology 6. DOI: 10.1126/sciimmunol.abg5669.

Mallapaty S. 2021. WHO approval of Chinese CoronaVac COVID vaccine will be crucial to curbing pandemic. Nature 594:161-162. DOI: 10.1038/d41586-021-01497-8.

Mallavarpu Ambrose J, Priya Veeraraghavan V, Kullappan M, Chellapandiyan P, Krishna Mohan S, Manivel VA. 2021. Comparison of Immunological Profiles of SARS-CoV-2 Variants in the COVID-19 Pandemic Trends: An Immunoinformatics Approach. Antibiotics (Basel, Switzerland) 10. DOI: 10.3390/antibiotics10050535.

Malonis RJ, Lai JR, Vergnolle O. 2020. Peptide-Based Vaccines: Current Progress and Future Challenges. Chemical reviews 120:3210-3229. DOI: 10.1021/acs.chemrev.9b00472.

Mascellino MT, Di Timoteo F, De Angelis M, Oliva A. 2021. Overview of the Main Anti-SARSCoV-2 Vaccines: Mechanism of Action, Efficacy and Safety. Individual differences research: IDR 14:3459-3476. DOI: 10.2147/IDR.S315727.

McGill COVID19 Vaccine Tracker Team. 2021.COVID-19 Vaccine Tracker. Available at https://covid19.trackvaccines.org/vaccines/\#approved (accessed September 2021).

McGuffin LJ, Bryson K, Jones DT. 2000. The PSIPRED protein structure prediction server. Bioinformatics 16:404-405. DOI: 10.1093/bioinformatics/16.4.404.

Medhi R, Srinoi P, Ngo N, Tran H-V, Lee TR. 2020. Nanoparticle-Based Strategies to Combat COVID-19. ACS Applied Nano Materials 3:8557-8580. DOI: 10.1021/acsanm.0c01978.

Menezes Teles E Oliveira D, Melo Santos de Serpa Brandão R, Claudio Demes da Mata Sousa L, das Chagas Alves Lima F, Jamil Hadad do Monte S, Sérgio Coelho Marroquim M, Vanildo de Sousa Lima A, Gilberto Borges Coelho A, Matheus Sousa Costa J, Martins Ramos R, Socorro da Silva A. 2019. pHLA3D: An online database of predicted three-dimensional 
882

883

884

885

886

887

888

889

890

891

892

893

894

895

896

897

898

899

900

901

902

903

904

905

906

907

908

909

910

911

912

913

914

915

916

917

918

919

920

921

922

923

924

925

926

927

928

929

930 structures of HLA molecules. Human immunology 80:834-841. DOI:

10.1016/j.humimm.2019.06.009.

Meyers LM, Gutiérrez AH, Boyle CM, Terry F, McGonnigal BG, Salazar A, Princiotta MF, Martin WD, De Groot AS, Moise L. 2021. Highly conserved, non-human-like, and crossreactive SARS-CoV-2 T cell epitopes for COVID-19 vaccine design and validation. NPJ vaccines 6:71. DOI: 10.1038/s41541-021-00331-6.

Minervina AA, Komech EA, Titov A, Bensouda Koraichi M, Rosati E, Mamedov IZ, Franke A, Efimov GA, Chudakov DM, Mora T, Walczak AM, Lebedev YB, Pogorelyy MV. 2021. Longitudinal high-throughput TCR repertoire profiling reveals the dynamics of T-cell memory formation after mild COVID-19 infection. eLife 10. DOI: 10.7554/eLife.63502.

Mohammad MG, Ibrahim F, Navid N, Shirin M. 2020. Multi-Epitope vaccines (MEVs), as a novel strategy against infectious diseases. Current proteomics 17:354-364.

Nagpal G, Chaudhary K, Agrawal P, Raghava GPS. 2018. Computer-aided prediction of antigen presenting cell modulators for designing peptide-based vaccine adjuvants. Journal of translational medicine 16:181. DOI: 10.1186/s12967-018-1560-1.

Nagpal G, Usmani SS, Dhanda SK, Kaur H, Singh S, Sharma M, Raghava GPS. 2017. Computer-aided designing of immunosuppressive peptides based on IL-10 inducing potential. Scientific reports 7:42851. DOI: 10.1038/srep42851.

Naveca FG, Nascimento V, de Souza VC, Corado A de L, Nascimento F, Silva G, Costa Á, Duarte D, Pessoa K, Mejía M, Brandão MJ, Jesus M, Gonçalves L, da Costa CF, Sampaio V, Barros D, Silva M, Mattos T, Pontes G, Abdalla L, Santos JH, Arantes I, Dezordi FZ, Siqueira MM, Wallau GL, Resende PC, Delatorre E, Gräf T, Bello G. 2021. COVID-19 in Amazonas, Brazil, was driven by the persistence of endemic lineages and P.1 emergence. Nature medicine. DOI: 10.1038/s41591-021-01378-7.

Naveed M, Tehreem S, Arshad S, Bukhari SA, Shabbir MA, Essa R, Ali N, Zaib S, Khan A, AlHarrasi A, Khan I. 2021. Design of a novel multiple epitope-based vaccine: An immunoinformatics approach to combat SARS-CoV-2 strains. Journal of infection and public health. DOI: 10.1016/j.jiph.2021.04.010.

Nemati AS, Tafrihi M, Sheikhi F, Tabari AR, Haditabar A. 2021. Designing a new multi epitopebased vaccine against COVID-19 disease: an immunoinformatic study based on reverse vaccinology approach. Research Square. DOI: 10.21203/rs.3.rs-206270/v1.

Ojha R, Gupta N, Naik B, Singh S, Verma VK, Prusty D, Prajapati VK. 2020. High throughput and comprehensive approach to develop multiepitope vaccine against minacious COVID-19. European journal of pharmaceutical sciences: official journal of the European Federation for Pharmaceutical Sciences 151:105375. DOI: 10.1016/j.ejps.2020.105375.

Oli AN, Obialor WO, Ifeanyichukwu MO, Odimegwu DC, Okoyeh JN, Emechebe GO, Adejumo SA, Ibeanu GC. 2020. Immunoinformatics and Vaccine Development: An Overview. ImmunoTargets and therapy 9:13-30. DOI: 10.2147/ITT.S241064.

O’Neill LAJ, Golenbock D, Bowie AG. 2013. The history of Toll-like receptors - redefining innate immunity. Nature reviews. Immunology 13:453-460. DOI: 10.1038/nri3446.

Ou X, Liu Y, Lei X, Li P, Mi D, Ren L, Guo L, Guo R, Chen T, Hu J, Xiang Z, Mu Z, Chen X, Chen J, Hu K, Jin Q, Wang J, Qian Z. 2020. Characterization of spike glycoprotein of SARS-CoV-2 on virus entry and its immune cross-reactivity with SARS-CoV. Nature communications 11:1620. DOI: 10.1038/s41467-020-15562-9.

Patra R, Chandra Das N, Mukherjee S. 2021. Targeting human TLRs to combat COVID-19: A solution? Journal of medical virology 93:615-617. DOI: 10.1002/jmv.26387.

Patronov A, Dimitrov I, Flower DR, Doytchinova I. 2011. Peptide binding prediction for the human class II MHC allele HLA-DP2: a molecular docking approach. BMC structural biology 11:32. DOI: 10.1186/1472-6807-11-32. 
931 Paul S, Lindestam Arlehamn CS, Scriba TJ, Dillon MBC, Oseroff C, Hinz D, McKinney DM,

932

933

934

935

936

937

938

939

940

941

942

943

944

945

946

947

948

949

950

951

952

953

954

955

956

957

958

959

960

961

962

963

964

965

966

967

968

969

970

971

972

973

974

975

976

977

978

979 Carrasco Pro S, Sidney J, Peters B, Sette A. 2015. Development and validation of a broad scheme for prediction of HLA class II restricted T cell epitopes. Journal of immunological methods 422:28-34. DOI: 10.1016/j.jim.2015.03.022.

Pham T-H, Qiu Y, Zeng J, Xie L, Zhang P. 2021. A deep learning framework for highthroughput mechanism-driven phenotype compound screening and its application to COVID-19 drug repurposing. Nature machine intelligence 3:247-257. DOI: 10.1038/s42256-020-00285-9.

Prachar M, Justesen S, Steen-Jensen DB, Thorgrimsen S, Jurgons E, Winther O, Bagger FO. 2020. Identification and validation of 174 COVID-19 vaccine candidate epitopes reveals low performance of common epitope prediction tools. Scientific reports 10:20465. DOI: 10.1038/ s41598-020-77466-4.

Prévost J, Finzi A. 2021. The great escape? SARS-CoV-2 variants evading neutralizing responses. Cell host \& microbe 29:322-324. DOI: 10.1016/j.chom.2021.02.010.

Quast I, Tarlinton D. 2021. B cell memory: understanding COVID-19. Immunity 54:205-210. DOI: 10.1016/j.immuni.2021.01.014.

Rahman MS, Hoque MN, Islam MR, Akter S, Rubayet Ul Alam ASM, Siddique MA, Saha O, Rahaman MM, Sultana M, Crandall KA, Hossain MA. 2020. Epitope-based chimeric peptide vaccine design against $\mathrm{S}, \mathrm{M}$ and $\mathrm{E}$ proteins of SARS-CoV-2, the etiologic agent of COVID19 pandemic: an in silico approach. PeerJ 8:e9572. DOI: 10.7717/peerj.9572.

Rakib A, Sami SA, Mimi NJ, Chowdhury MM, Eva TA, Nainu F, Paul A, Shahriar A, Tareq AM, Emon NU, Chakraborty S, Shil S, Mily SJ, Ben Hadda T, Almalki FA, Emran TB. 2020. Immunoinformatics-guided design of an epitope-based vaccine against severe acute respiratory syndrome coronavirus 2 spike glycoprotein. Computers in biology and medicine 124:103967. DOI: 10.1016/j.compbiomed.2020.103967.

Ramana J, Mehla K. 2020. Immunoinformatics and Epitope Prediction. In: Tomar N ed. Immunoinformatics. New York, NY: Springer US, 155-171. DOI: 10.1007/978-1-07160389-5_6.

Rambaut A, Loman N, Pybus O, Barclay W, Barrett J, Carabelli A, Connor T, Peacock T, Robertson DL, Volz E. 2020.Preliminary genomic characterisation of an emergent SARS$\mathrm{CoV}-2$ lineage in the UK defined by a novel set of spike mutations. Available at https://virological.org/t/preliminary-genomic-characterisation-of-an-emergent-sars-cov-2lineage-in-the-uk-defined-by-a-novel-set-of-spike-mutations/563 (accessed June 2021).

Rapin N, Lund O, Bernaschi M, Castiglione F. 2010. Computational immunology meets bioinformatics: the use of prediction tools for molecular binding in the simulation of the immune system. PloS one 5:e9862. DOI: 10.1371/journal.pone.0009862.

Redd AD, Nardin A, Kared H, Bloch EM, Pekosz A, Laeyendecker O, Abel B, Fehlings M, Quinn TC, Tobian AA. 2021. CD8+ T cell responses in COVID-19 convalescent individuals target conserved epitopes from multiple prominent SARS-CoV-2 circulating variants. medRxiv : the preprint server for health sciences. DOI: 10.1101/2021.02.11.21251585.

Ribes M, Chaccour C, Moncunill G. 2021. Adapt or perish: SARS-CoV-2 antibody escape variants defined by deletions in the Spike N-terminal Domain. Signal transduction and targeted therapy 6:164. DOI: 10.1038/s41392-021-00601-8.

Rotondo JC, Martini F, Maritati M, Mazziotta C, Di Mauro G, Lanzillotti C, Barp N, Gallerani A, Tognon M, Contini C. 2021. SARS-CoV-2 Infection: New Molecular, Phylogenetic, and Pathogenetic Insights. Efficacy of Current Vaccines and the Potential Risk of Variants. Viruses 13:1687. DOI: 10.3390/v13091687.

Saad-Roy CM, Morris SE, Metcalf CJE, Mina MJ, Baker RE, Farrar J, Holmes EC, Pybus OG, Graham AL, Levin SA, Grenfell BT, Wagner CE. 2021. Epidemiological and evolutionary

Peer) reviewing PDF | (2021:06:63076:2:0:NEW 4 Nov 2021) 
980

981

982

983

984

985

986

987

988

989

990

991

992

993

994

995

996

997

998

999

1000

1001

1002

1003

1004

1005

1006

1007

1008

1009

1010

1011

1012

1013

1014

1015

1016

1017

1018

1019

1020

1021

1022

1023

1024

1025

1026

1027

1028

considerations of SARS-CoV-2 vaccine dosing regimes. Science 372:363-370. DOI: 10.1126/science.abg8663.

Saba AA, Adiba M, Saha P, Hosen MI, Chakraborty S, Nabi AHMN. 2021. An in-depth in silico and immunoinformatics approach for designing a potential multi-epitope construct for the effective development of vaccine to combat against SARS-CoV-2 encompassing variants of concern and interest. Computers in biology and medicine 136:104703. DOI:

10.1016/j.compbiomed.2021.104703.

Sakaguchi T, Ibe M, Miwa K, Kaneko Y, Yokota S, Tanaka K, Takiguchi M. 1997. Binding of 8mer to 11-mer peptides carrying the anchor residues to slow assembling HLA class I molecules (HLA-B*5101). Immunogenetics 45:259-265. DOI: 10.1007/s002510050201.

Sarma VR, Olotu FA, Soliman MES. 2021. Integrative immunoinformatics paradigm for predicting potential B-cell and T-cell epitopes as viable candidates for subunit vaccine design against COVID-19 virulence. Biomedical journal. DOI: 10.1016/j.bj.2021.05.001.

Sette A, Crotty S. 2021. Adaptive immunity to SARS-CoV-2 and COVID-19. Cell 184:861-880. DOI: 10.1016/j.cell.2021.01.007.

Shahcheraghi SH, Ayatollahi J, Aljabali AA, Shastri MD, Shukla SD, Chellappan DK, Jha NK, Anand K, Katari NK, Mehta M, Satija S, Dureja H, Mishra V, Almutary AG, Alnuqaydan AM, Charbe N, Prasher P, Gupta G, Dua K, Lotfi M, Bakshi HA, Tambuwala MM. 2021. An overview of vaccine development for COVID-19. Therapeutic delivery 12:235-244. DOI: 10.4155/tde-2020-0129.

Shang W, Yang Y, Rao Y, Rao X. 2020. The outbreak of SARS-CoV-2 pneumonia calls for viral vaccines. NPJ vaccines 5:18. DOI: 10.1038/s41541-020-0170-0.

Sharma A, Pal S, Panwar A, Kumar S, Kumar A. 2021. In-silico immunoinformatic analysis of SARS-CoV-2 virus for the development of putative vaccine construct. Immunobiology 226:152134. DOI: 10.1016/j.imbio.2021.152134.

Sharma N, Patiyal S, Dhall A, Pande A, Arora C, Raghava GPS. 2020. AlgPred 2.0: an improved method for predicting allergenic proteins and mapping of IgE epitopes. Briefings in bioinformatics. DOI: 10.1093/bib/bbaa294.

Shean RC, Makhsous N, Stoddard GD, Lin MJ, Greninger AL. 2019. VAPiD: a lightweight cross-platform viral annotation pipeline and identification tool to facilitate virus genome submissions to NCBI GenBank. BMC bioinformatics 20:1-8. DOI: 10.1186/s12859-0192606-y.

Shkurnikov M, Nersisyan S, Jankevic T, Galatenko A, Gordeev I, Vechorko V, Tonevitsky A. 2021. Association of HLA Class I Genotypes With Severity of Coronavirus Disease-19. Frontiers in immunology 12:641900. DOI: 10.3389/fimmu.2021.641900.

Sievers F, Higgins DG. 2018. Clustal Omega for making accurate alignments of many protein sequences. Protein science: a publication of the Protein Society 27:135-145. DOI: 10.1002/ pro.3290.

Singh A, Thakur M, Sharma LK, Chandra K. 2020. Designing a multi-epitope peptide based vaccine against SARS-CoV-2. Scientific reports 10:16219. DOI: 10.1038/s41598-02073371-y.

Siracusano G, Pastori C, Lopalco L. 2020. Humoral Immune Responses in COVID-19 Patients: A Window on the State of the Art. Frontiers in immunology 11:1049. DOI: 10.3389/fimmu.2020.01049.

Skwarczynski M, Toth I. 2016. Peptide-based synthetic vaccines. Chemical science 7:842-854. DOI: $10.1039 / \mathrm{c} 5 \mathrm{sc} 03892 \mathrm{~h}$.

Sun L. 2013. Peptide-Based Drug Development. Modern Chemistry and Applications 1. DOI: 10.4172/mca.1000e103.

Tarke A, Sidney J, Methot N, Yu ED, Zhang Y, Dan JM, Goodwin B, Rubiro P, Sutherland A, 
1029

1030

1031

1032

1033

1034

1035

1036

1037

1038

1039

1040

1041

1042

1043

1044

1045

1046

1047

1048

1049

1050

1051

1052

1053

1054

1055

1056

1057

1058

1059

1060

1061

1062

1063

1064

1065

1066

1067

1068

1069

1070

1071

1072

1073

1074

1075

1076

1077

Wang E, Frazier A, Ramirez SI, Rawlings SA, Smith DM, da Silva Antunes R, Peters B, Scheuermann RH, Weiskopf D, Crotty S, Grifoni A, Sette A. 2021. Impact of SARS-CoV-2 variants on the total CD4+ and CD8+ T cell reactivity in infected or vaccinated individuals. Cell reports. Medicine 2:100355. DOI: 10.1016/j.xcrm.2021.100355.

Tavasolian F, Rashidi M, Hatam GR, Jeddi M, Hosseini AZ, Mosawi SH, Abdollahi E, Inman RD. 2020. HLA, Immune Response, and Susceptibility to COVID-19. Frontiers in immunology 11:601886. DOI: 10.3389/fimmu.2020.601886.

Tegally H, Wilkinson E, Giovanetti M, Iranzadeh A, Fonseca V, Giandhari J, Doolabh D, Pillay S, San EJ, Msomi N, Mlisana K, von Gottberg A, Walaza S, Allam M, Ismail A, Mohale T, Glass AJ, Engelbrecht S, Van Zyl G, Preiser W, Petruccione F, Sigal A, Hardie D, Marais G, Hsiao N-Y, Korsman S, Davies M-A, Tyers L, Mudau I, York D, Maslo C, Goedhals D, Abrahams S, Laguda-Akingba O, Alisoltani-Dehkordi A, Godzik A, Wibmer CK, Sewell BT, Lourenço J, Alcantara LCJ, Kosakovsky Pond SL, Weaver S, Martin D, Lessells RJ, Bhiman JN, Williamson C, de Oliveira T. 2021. Detection of a SARS-CoV-2 variant of concern in South Africa. Nature 592:438-443. DOI: 10.1038/s41586-021-03402-9.

Thanh Le T, Andreadakis Z, Kumar A, Gómez Román R, Tollefsen S, Saville M, Mayhew S. 2020. The COVID-19 vaccine development landscape. Nature reviews. Drug discovery 19:305-306. DOI: 10.1038/d41573-020-00073-5.

Thomson EC, Rosen LE, Shepherd JG, Spreafico R, da Silva Filipe A, Wojcechowskyj JA, Davis C, Piccoli L, Pascall DJ, Dillen J, Lytras S, Czudnochowski N, Shah R, Meury M, Jesudason N, De Marco A, Li K, Bassi J, O’Toole A, Pinto D, Colquhoun RM, Culap K, Jackson B, Zatta F, Rambaut A, Jaconi S, Sreenu VB, Nix J, Zhang I, Jarrett RF, Glass WG, Beltramello M, Nomikou K, Pizzuto M, Tong L, Cameroni E, Croll TI, Johnson N, Di Iulio J, Wickenhagen A, Ceschi A, Harbison AM, Mair D, Ferrari P, Smollett K, Sallusto F, Carmichael S, Garzoni C, Nichols J, Galli M, Hughes J, Riva A, Ho A, Schiuma M, Semple MG, Openshaw PJM, Fadda E, Baillie JK, Chodera JD, ISARIC4C Investigators, COVID19 Genomics UK (COG-UK) Consortium, Rihn SJ, Lycett SJ, Virgin HW, Telenti A, Corti D, Robertson DL, Snell G. 2021. Circulating SARS-CoV-2 spike N439K variants maintain fitness while evading antibody-mediated immunity. Cell 184:1171-1187.e20. DOI: 10.1016/ j.cell.2021.01.037.

Tomita Y, Ikeda T, Sato R, Sakagami T. 2020. Association between HLA gene polymorphisms and mortality of COVID-19: An in silico analysis. Immunity, inflammation and disease 8:684-694. DOI: 10.1002/iid3.358.

Vita R, Mahajan S, Overton JA, Dhanda SK, Martini S, Cantrell JR, Wheeler DK, Sette A, Peters B. 2019. The Immune Epitope Database (IEDB): 2018 update. Nucleic acids research 47:D339-D343. DOI: 10.1093/nar/gky1006.

Walls AC, Park Y-J, Tortorici MA, Wall A, McGuire AT, Veesler D. 2020. Structure, Function, and Antigenicity of the SARS-CoV-2 Spike Glycoprotein. Cell 181:281-292.e6. DOI: 10.1016/j.cell.2020.02.058.

Wang X, Gui J. 2020. Cell-mediated immunity to SARS-CoV-2. Pediatric investigation 4:281291. DOI: $10.1002 /$ ped4.12228.

WHO. 2021.Weekly epidemiological update on COVID-19 - 25 May 2021. Available at https:// www.who.int/publications/m/item/weekly-epidemiological-update-on-covid-19---25-may2021 (accessed May 27, 2021).

Wiederstein M, Sippl MJ. 2007. ProSA-web: interactive web service for the recognition of errors in three-dimensional structures of proteins. Nucleic acids research 35:W407-10. DOI: 10.1093/nar/gkm290.

Wu D, Yang XO. 2020. TH17 responses in cytokine storm of COVID-19: An emerging target of JAK2 inhibitor Fedratinib. Journal of microbiology, immunology, and infection = Wei mian

Peer) reviewing PDF | (2021:06:63076:2:0:NEW 4 Nov 2021) 
1078

1079

1080

1081

1082

1083

1084

1085

1086

1087

1088

1089

1090

1091

1092

1093

1094

1095

1096

1097

1098

1099

1100

1101

1102

1103

1104 yu gan ran za zhi 53:368-370. DOI: 10.1016/j.jmii.2020.03.005.

Xue LC, Rodrigues JP, Kastritis PL, Bonvin AM, Vangone A. 2016. PRODIGY: a web server for predicting the binding affinity of protein-protein complexes. Bioinformatics 32:3676-3678. DOI: 10.1093/bioinformatics/btw514.

Yang Z, Bogdan P, Nazarian S. 2021. An in silico deep learning approach to multi-epitope vaccine design: a SARS-CoV-2 case study. Scientific reports 11:3238. DOI: 10.1038/s41598-021-81749-9.

Yang J, Wang W, Chen Z, Lu S, Yang F, Bi Z, Bao L, Mo F, Li X, Huang Y, Hong W, Yang Y, Zhao Y, Ye F, Lin S, Deng W, Chen H, Lei H, Zhang Z, Luo M, Gao H, Zheng Y, Gong Y, Jiang X, Xu Y, Lv Q, Li D, Wang M, Li F, Wang S, Wang G, Yu P, Qu Y, Yang L, Deng H, Tong A, Li J, Wang Z, Yang J, Shen G, Zhao Z, Li Y, Luo J, Liu H, Yu W, Yang M, Xu J, Wang J, Li H, Wang H, Kuang D, Lin P, Hu Z, Guo W, Cheng W, He Y, Song X, Chen C, Xue Z, Yao S, Chen L, Ma X, Chen S, Gou M, Huang W, Wang Y, Fan C, Tian Z, Shi M, Wang F-S, Dai L, Wu M, Li G, Wang G, Peng Y, Qian Z, Huang C, Lau JY-N, Yang Z, Wei Y, Cen X, Peng X, Qin C, Zhang K, Lu G, Wei X. 2020. A vaccine targeting the RBD of the $S$ protein of SARS-CoV-2 induces protective immunity. Nature 586:572-577. DOI: 10.1038/s41586-020-2599-8.

Yoshida S, Ono C, Hayashi H, Fukumoto S, Shiraishi S, Tomono K, Arase H, Matsuura Y, Nakagami H. 2021. SARS-CoV-2-induced humoral immunity through B cell epitope analysis in COVID-19 infected individuals. Scientific reports 11:5934. DOI: 10.1038/s41598-021-85202-9.

Zaheer T, Waseem M, Waqar W, Dar HA, Shehroz M, Naz K, Ishaq Z, Ahmad T, Ullah N, Bakhtiar SM, Muhammad SA, Ali A. 2020. Anti-COVID-19 multi-epitope vaccine designs employing global viral genome sequences. PeerJ 8:e9541. DOI: 10.7717/peerj.9541.

Zheng M, Song L. 2020. Novel antibody epitopes dominate the antigenicity of spike glycoprotein in SARS-CoV-2 compared to SARS-CoV. Cellular \& molecular immunology 17:536-538. DOI: $10.1038 /$ s41423-020-0385-z. 


\section{Figure 1}

Schematic overview of the prediction, accurate selection and epitopes validation to identify new SARS-CoV-2 epitopes.

The pipeline comprises four main analyses: prediction and curation epitopes selection, immune properties evaluation and Selected epitopes validation. (A) The prediction encompasses three DTU Health Tech software (blue boxes) subdivided according to the type of identified epitopes: CD8 + T cell, CD4 + T cell and B cell epitopes. (B) The curation step uses the predicted epitopes for an accurate selection comprising our proposal EpiCurator workflow (pink boxes) and online server to evaluate the antigenicity, toxicity and allergen analysis. (C) The pipeline has a set of individual analyses that identify the population coverage, immunogenicity and other immunogenic properties using on-line servers (yellow boxes). (D) Additionally, a in silico validation of final RBD selected epitopes has been performed using a multi-epitope construct. Their linear, 2D and 3D sequences are evaluated using on-line servers (yellow boxes) to characterize the multi-epitope and the construct-TLR complexes as stable and immunogenic. Each analysis has its respective parameters presented below the boxes. 


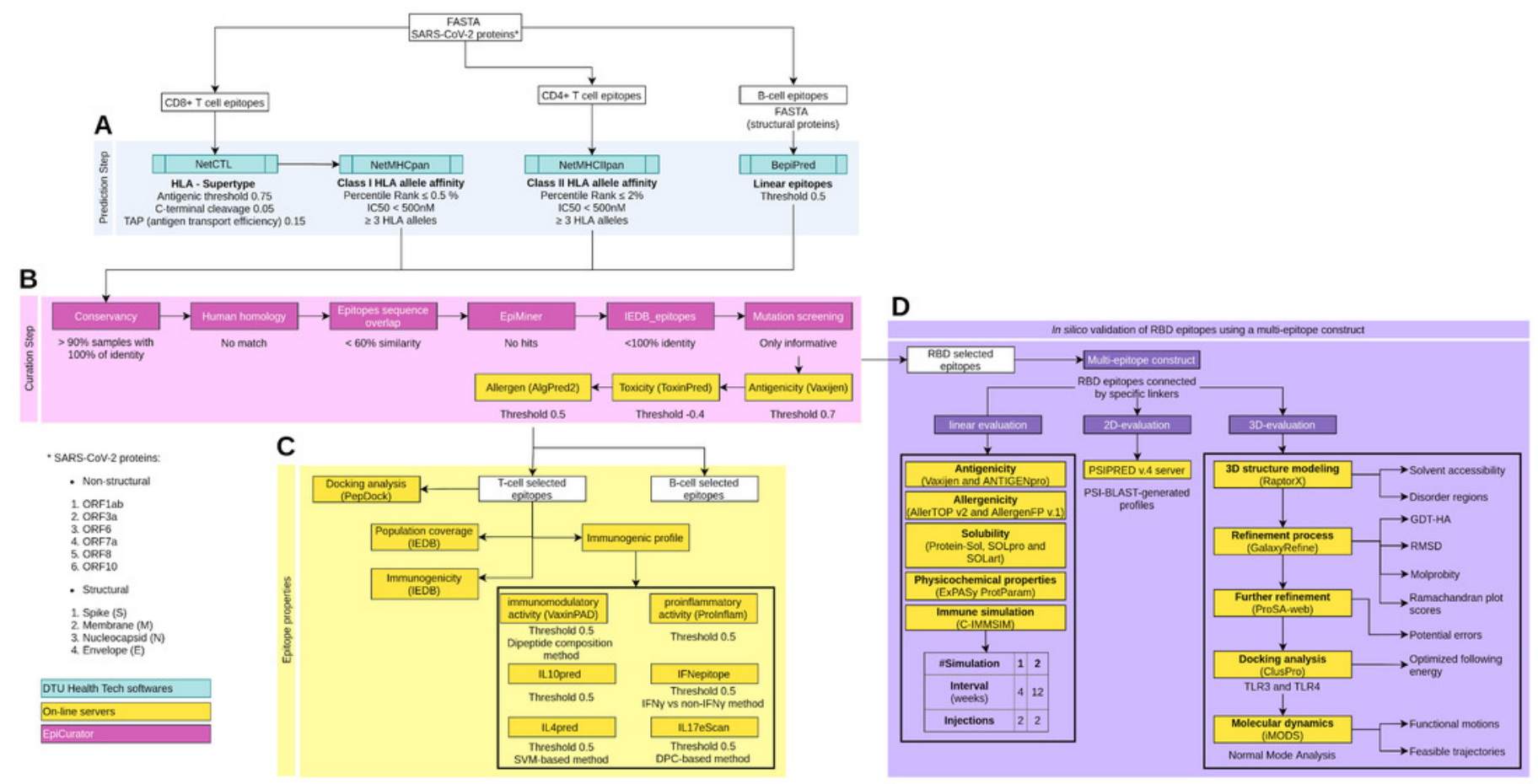




\section{Figure 2}

Efficiency of EpiCurator analysis for accurate selection of epitopes.

The plot represents the percentage of epitopes removed in each different analysis.
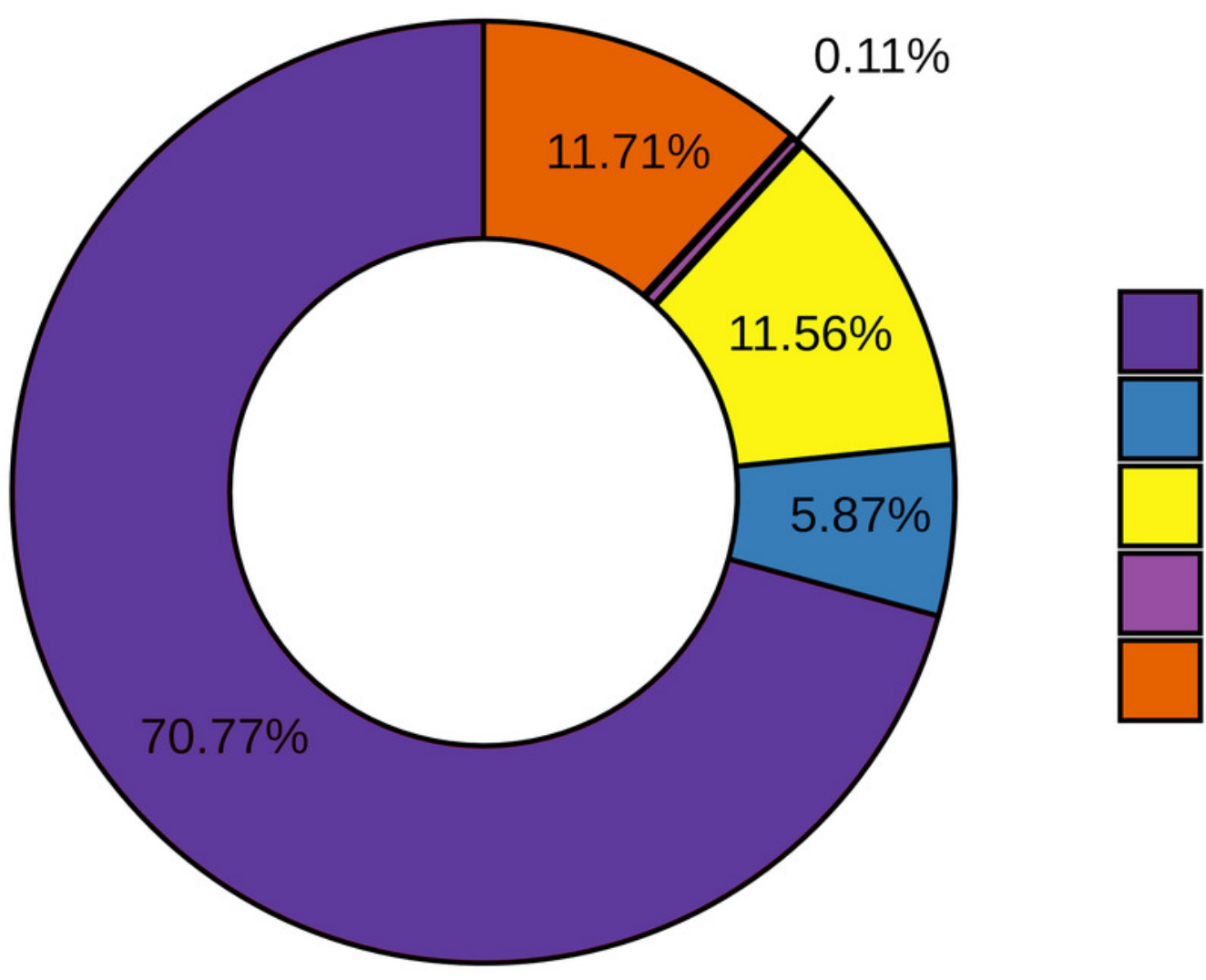

Conservancy

EpiMiner

Epitopes overlap

Human homology

IEDB matching 


\section{Figure 3}

Identification of final SARS-CoV-2-derived HLA class I- and class II-binding epitopes.

(A) Distribution of the accurately selected epitopes in the SARS-CoV-2 proteins. Each color represents a distinct protein. (B) Distribution of the accurately selected epitopes in each current Brazilian lineage. Each color represents a distinct epitope type. (C) Distribution of epitopes in HLA class I alleles. The color degree represents the variation of the number of epitopes. (D) Distribution of epitopes in HLA class II alleles. The color degree represents the variation of the number of epitopes.

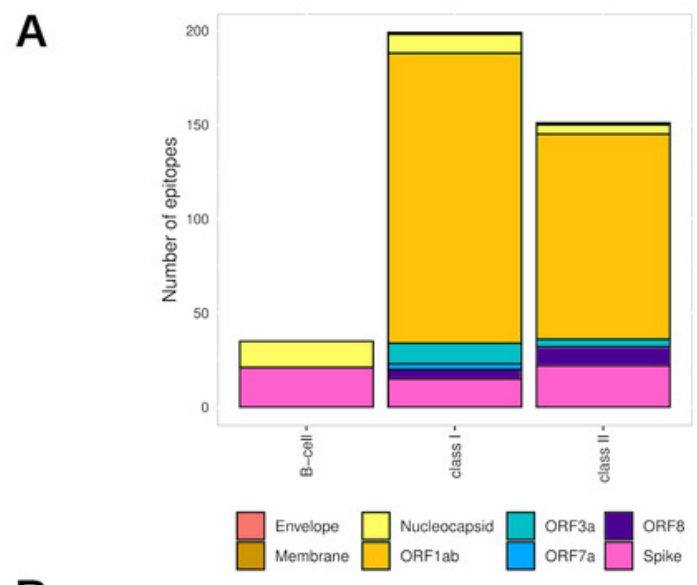

B

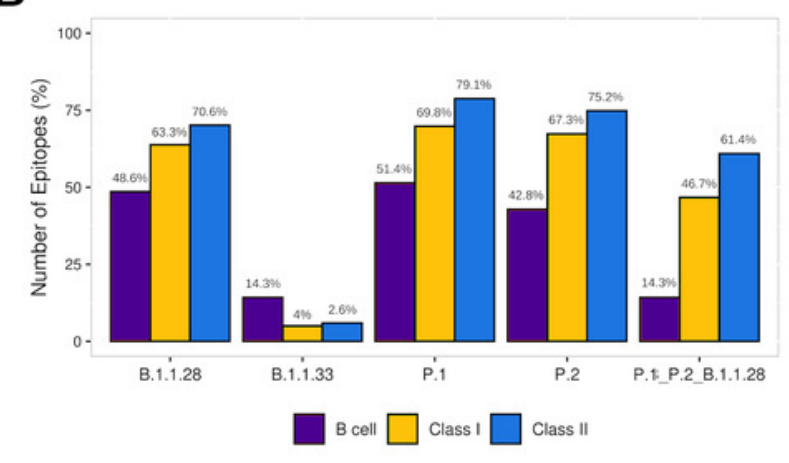

C

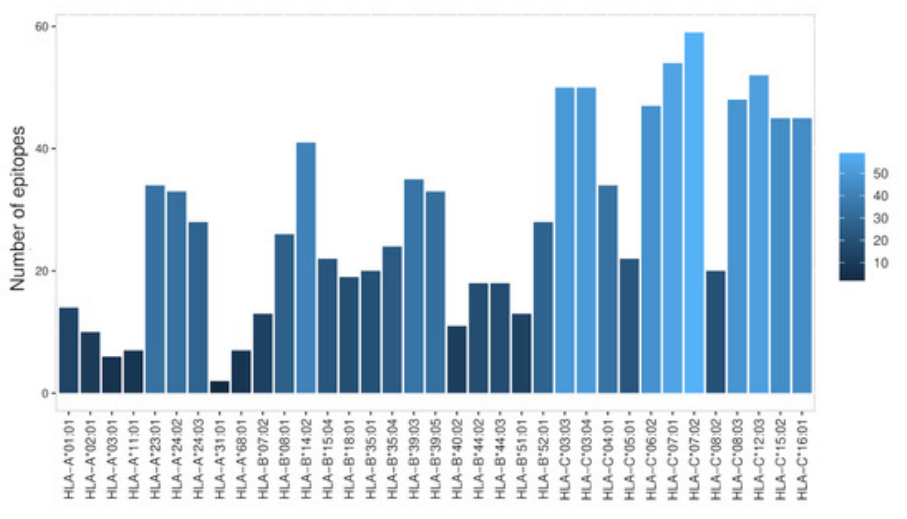

D

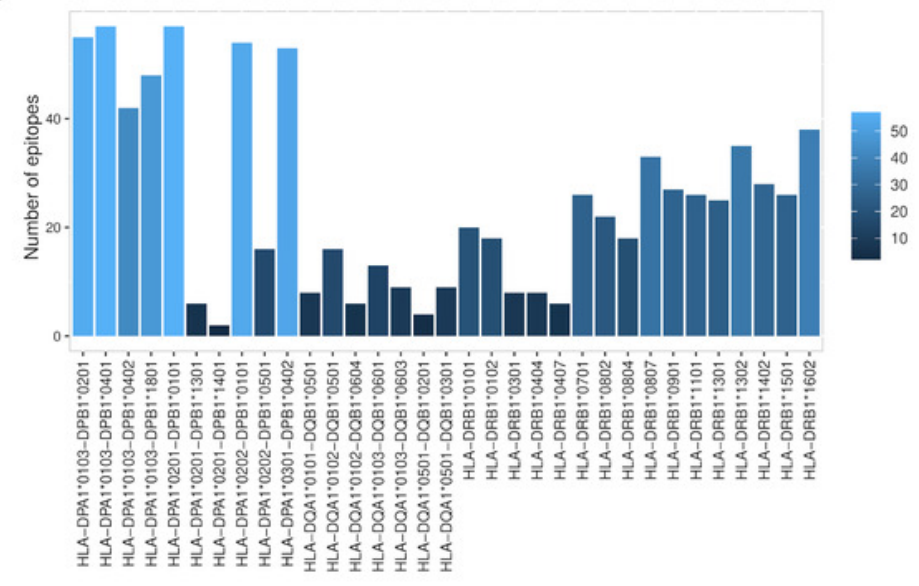




\section{Figure 4}

Distribution of the accurate selected epitopes in the structure of SARS-CoV-2 Spike glycoprotein.

Representation of Spike protein structure and their main domains. The epitopes are distributed in the Spike structure by their coordinate in the protein sequence allowing the identification of the domains where the epitopes were found. The specific sequence and coordinate of the epitopes found in the RBD domain are shown. The epitopes are colored by their type (Class I, Class II and B-cell epitopes).

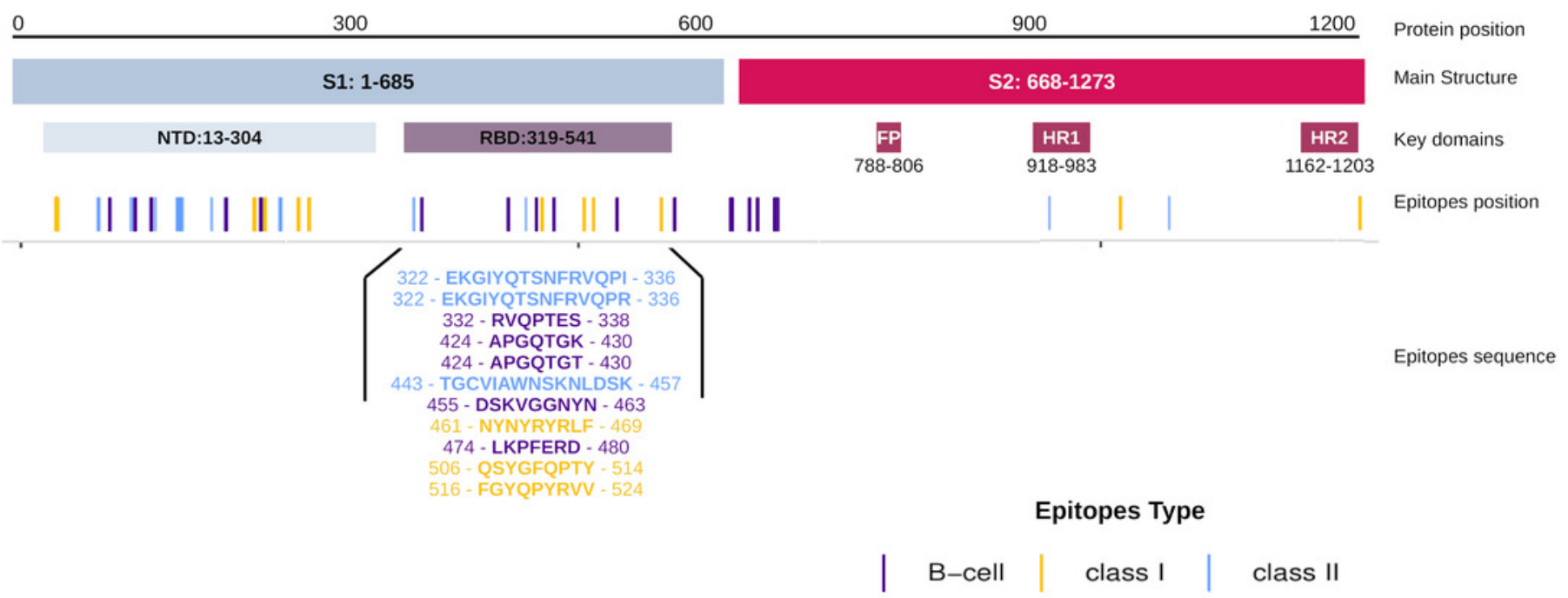

NTD - N-Terminal domain; RBD - Receptor Binding domain; FP - Fusion peptide; HR1 - Heptad repeat 1; HR2 - Heptad repeat 2 


\section{Figure 5}

Properties of epitopes from Spike RBD domain.

(A) The affinity values of the selected epitopes ( $x$ axis) are indicated as bars on the right $y$ axis, and the antigenicity values are indicated as dots on the left $y$ axis. (B) The number of HLA binding alleles for the selected epitopes ( $x$ axis) are indicated as bars on the left $y$ axis, and the cumulative percentage of population coverage is depicted as dots on the right $y$ axis. Each color of bars represents a distinct epitope type and each color of lines represents a distinct population.

A

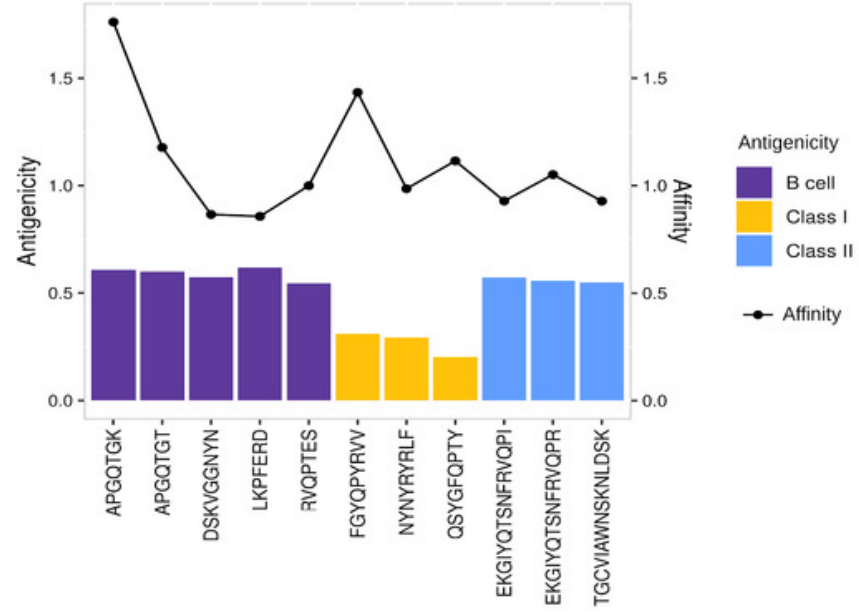

B

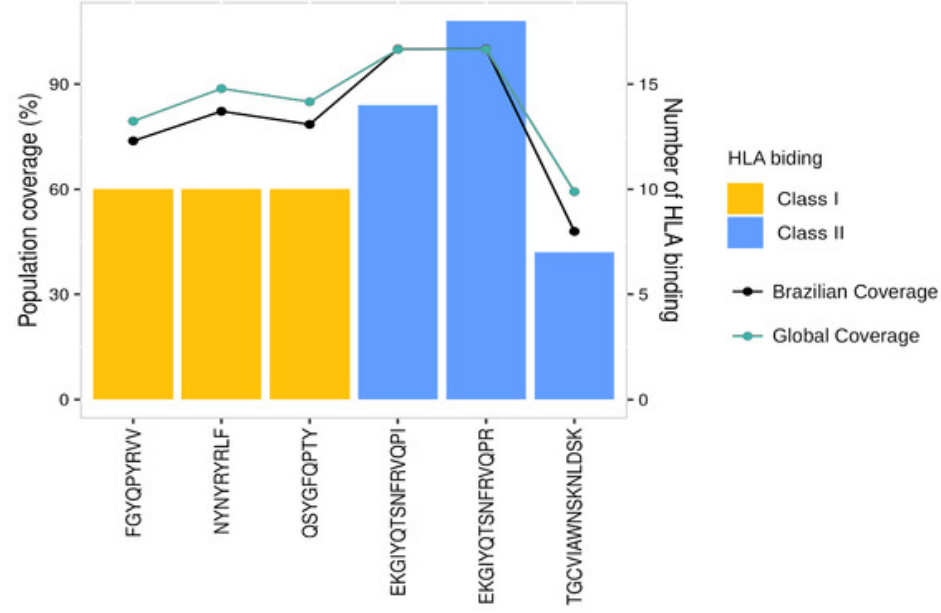


Figure 6

Structure of the HLA-epitope complex for the main T-cell epitopes in Spike RBD domain.

Structure complexes provided by docking simulation shows the MHC binding grooves (blue ribbons), and the epitope (red structure). For each complex, the amino acids sequence of the epitope, HLA binding allele and the binding free energy are available.

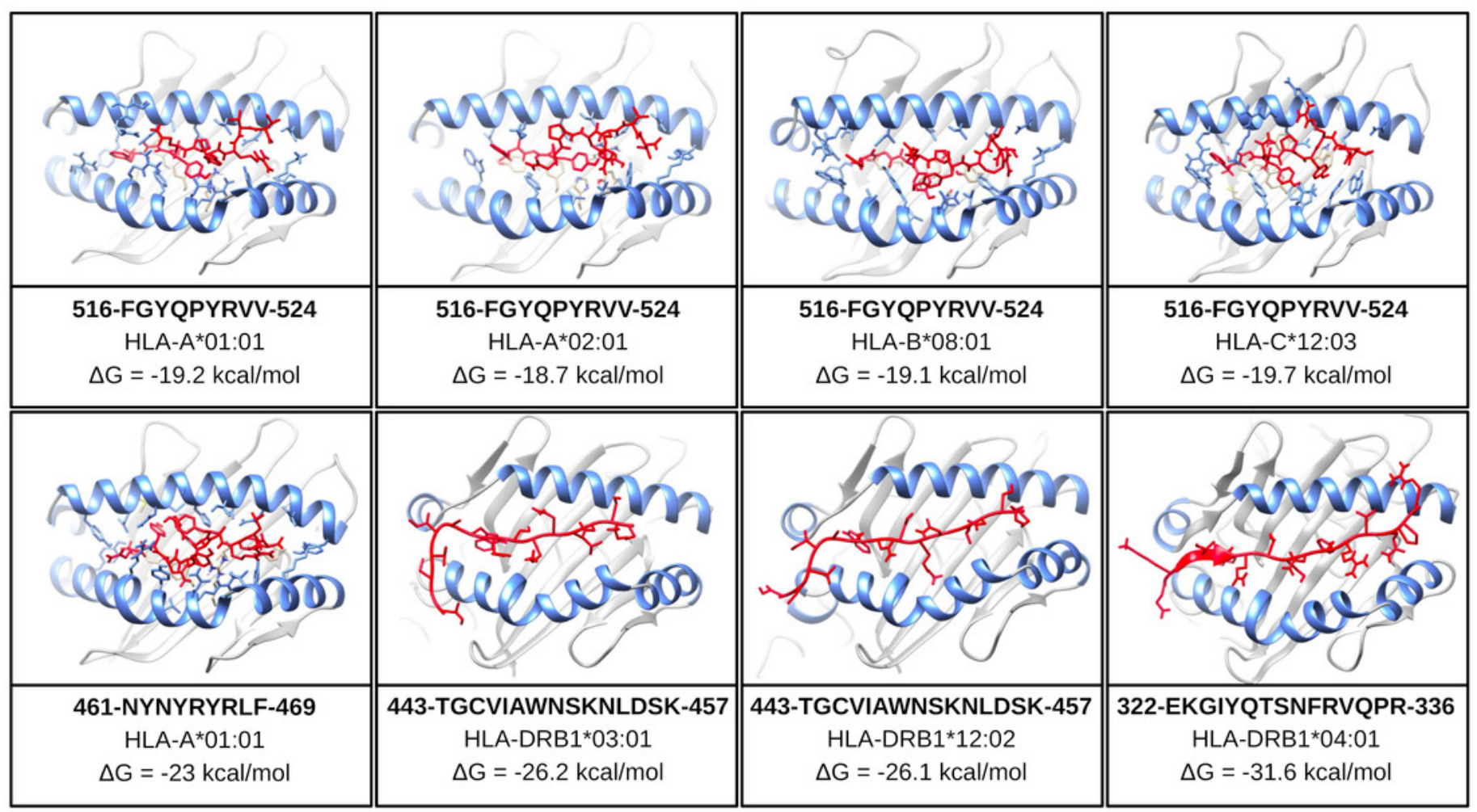




\section{Figure 7}

In silico simulation of immune response using multi-epitope construct as an antigen.

The multi-epitopes injection occurs on day 1 and within a 6-month interval. (A) Evolution of Th0, Th1, Th2 and Th17 response in cell/mm3 and percentage across the days. (B) Cytokine production across the days - specific subclasses are indicated as colored peaks. (C) multiepitope injections (black vertical lines) promoting the immunoglobulin production - specific subclasses are indicated as colored peaks.

A
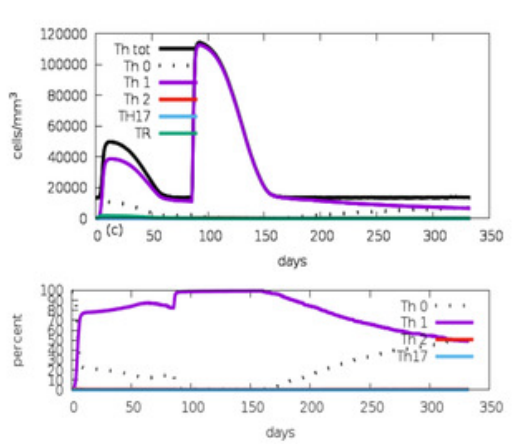

B

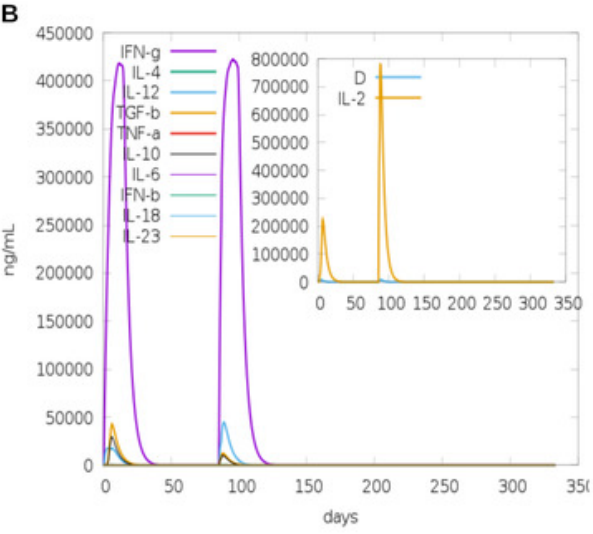

C

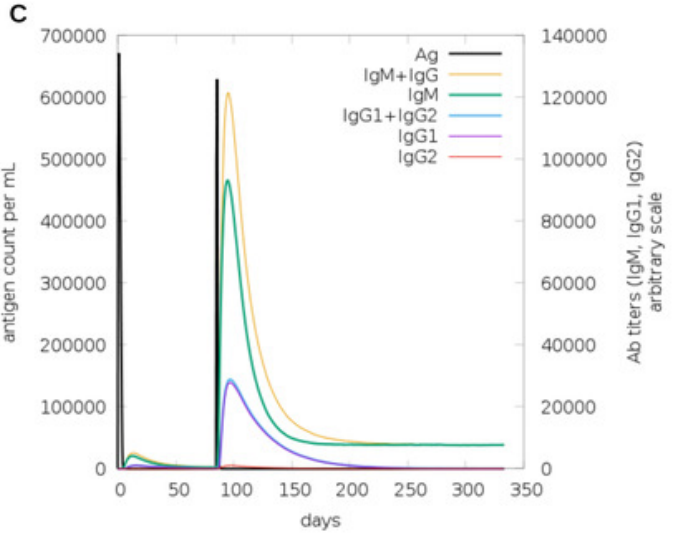




\section{Figure 8}

Structure of the multi-epitope-TLR complexes.

The immune receptors TLR3 and TLR4 are demonstrated in gray color and the ligands (multiepitope) are shown in pink color. (A) The multi-epitope-TLR3 complex (B) the multi-epitopeTLR4 complex.

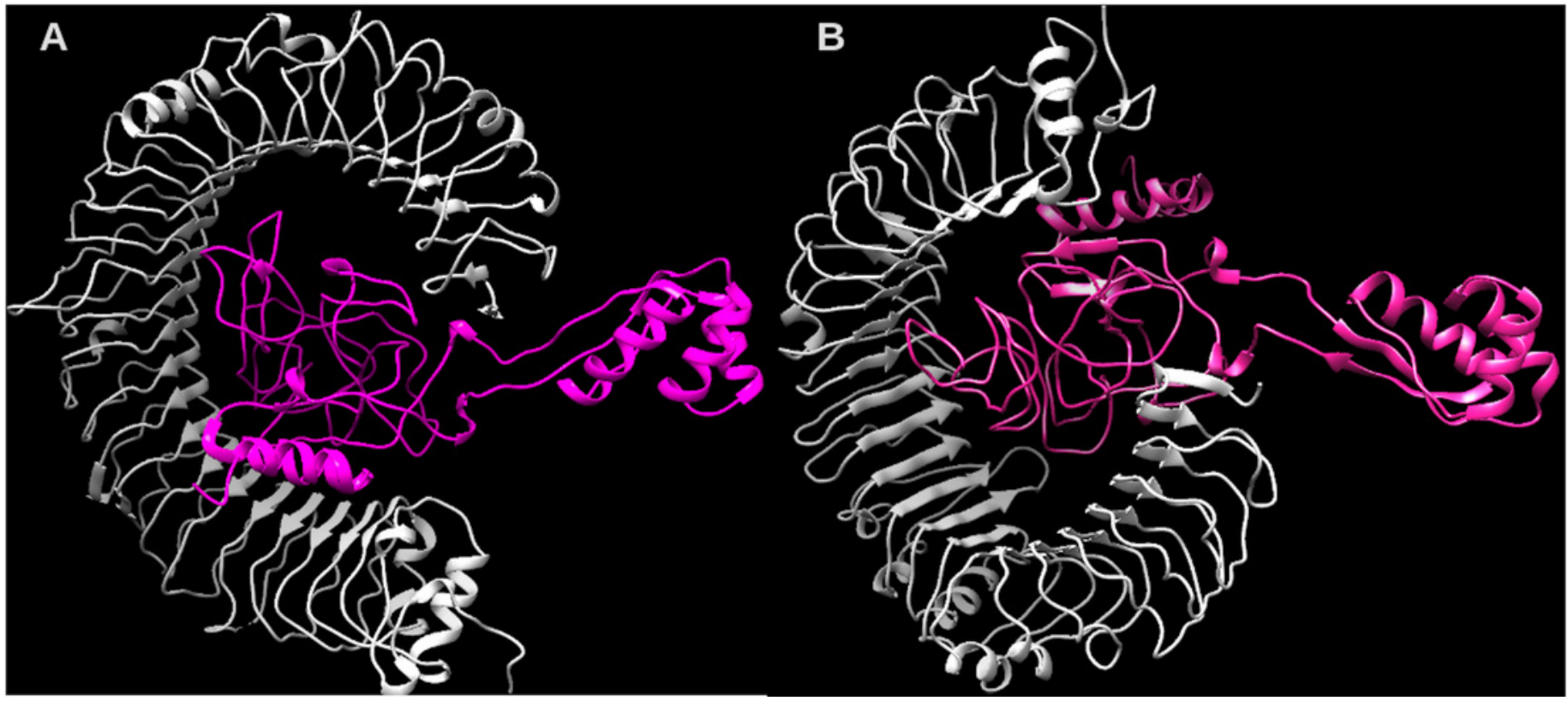




\section{Table $\mathbf{1}$ (on next page)}

Summary of SARS-CoV-2-derived epitopes predicted with high binding affinity to each HLA loci. 
1

\begin{tabular}{|c|c|c|c|c|c|c|c|c|c|c|}
\hline \multirow{3}{*}{ Protein } & \multirow{3}{*}{$\begin{array}{l}\text { Protein } \\
\text { length } \\
\text { (amino acids) }\end{array}$} & \multicolumn{9}{|c|}{ Number of epitopes } \\
\hline & & \multicolumn{4}{|c|}{$\begin{array}{l}\text { HLA class I-restricted T cell } \\
\text { epitopes }(\operatorname{rank} \leq 0.5)\end{array}$} & \multicolumn{4}{|c|}{$\begin{array}{l}\text { HLA class II-restricted T cell } \\
\text { epitopes }(\operatorname{rank} \leq 2)\end{array}$} & \multirow{2}{*}{$\begin{array}{l}\text { B cell } \\
\text { epitopes } \\
\text { All }\end{array}$} \\
\hline & & All & HLA-A & HLA-B & HLA-C & All & HLA-DP & HLA-DQ & HLA-DR & \\
\hline Envelope & 75 & 33 & 22 & 26 & 69 & 54 & - & - & 13 & 10 \\
\hline Membrane & 222 & 99 & 104 & 55 & 127 & 83 & 6 & 6 & 44 & 7 \\
\hline Nucleocapsid & 419 & 226 & 143 & 205 & 217 & 390 & 21 & 22 & 202 & 147 \\
\hline ORF10 & 38 & 23 & 12 & 12 & 29 & 10 & - & 1 & 8 & - \\
\hline ORF1ab & 7096 & 3621 & 2454 & 3517 & 4262 & 5225 & 1291 & 630 & 2608 & - \\
\hline ORF3a & 275 & 343 & 256 & 298 & 435 & 357 & 46 & 24 & 260 & - \\
\hline ORF6 & 61 & 32 & 15 & 20 & 26 & 47 & 13 & 4 & 24 & - \\
\hline ORF 7a & 121 & 96 & 65 & 110 & 96 & 102 & 101 & 2 & 71 & - \\
\hline ORF7b & 43 & 15 & 9 & 7 & 15 & 3 & - & - & 1 & - \\
\hline ORF8 & 121 & 98 & 51 & 87 & 113 & 139 & 57 & 8 & 35 & - \\
\hline Spike & 1273 & 675 & 427 & 652 & 775 & 1239 & 531 & 120 & 436 & 93 \\
\hline TOTAL & - & 5261 & 3558 & 4989 & 6164 & 7649 & 2066 & 817 & 3702 & 257 \\
\hline
\end{tabular}

2 


\section{Table 2 (on next page)}

Summary of final SARS-CoV-2-derived epitopes accurately selected to each SARS-CoV-2 protein. 
1

\begin{tabular}{lcccccc} 
& \multicolumn{2}{l}{$\begin{array}{l}\text { HLA class I-restricted T cell } \\
\text { epitopes }\end{array}$} & $\begin{array}{l}\text { HLA class II-restricted T cell } \\
\text { epitopes }\end{array}$ & B cell epitopes & \\
\hline Protein & $\begin{array}{c}\text { Prediction } \\
(\mathbf{n})\end{array}$ & $\begin{array}{c}\text { Curation } \\
(\mathbf{n}-\mathbf{\%})^{*}\end{array}$ & $\begin{array}{c}\text { Prediction } \\
(\mathbf{n})\end{array}$ & $\begin{array}{c}\text { Curation } \\
(\mathbf{n}-\mathbf{\%})^{*}\end{array}$ & $\begin{array}{c}\text { Prediction } \\
(\mathbf{n})\end{array}$ & $\begin{array}{c}\text { Curation } \\
(\mathbf{n}-\mathbf{\%})^{*}\end{array}$ \\
\hline Envelope & 33 & - & 54 & $1(1.85)$ & 10 & $1(10)$ \\
Membrane & 99 & $1(1.01)$ & 83 & - & 7 & - \\
Nucleocapsid & 226 & $10(4.25)$ & 390 & $5(1.28)$ & 147 & $14(9.52)$ \\
ORF10 & 23 & - & 10 & - & - & - \\
ORF1ab & 3621 & $154(4.25)$ & 5225 & $111(2.12)$ & - & - \\
ORF3a & 343 & $11(3.21)$ & 357 & $4(1.12)$ & - & - \\
ORF6 & 32 & - & 47 & - & - & - \\
ORF7a & 96 & $3(3.12)$ & 102 & - & - & - \\
ORF7b & 15 & - & 3 & - & - & - \\
ORF8 & 98 & $5(5.10)$ & 139 & $10(7.19)$ & - & $21(22.58)$ \\
Spike & 675 & $15(2.22)$ & 1239 & $22(1.77)$ & 93 & $\mathbf{3 6}(\mathbf{1 4 . 0 1 )}$ \\
\hline TOTAL & $\mathbf{5 2 6 1}$ & $\mathbf{1 9 9}(\mathbf{3 . 7 8 )}$ & $\mathbf{7 6 4 9}$ & $\mathbf{1 5 3} \mathbf{( 2 . 0 0 )}$ & $\mathbf{2 5 7}$ & \\
\hline
\end{tabular}

* Percentage related to number of predicted epitope per protein 